\title{
Long-range correlation and nonlinearity in geochemical time series of gas discharges from Mt. Etna, and changes with 2001 and 2002-2003 eruptions
}

\author{
A. Paonita \\ Istituto Nazionale di Geofisica e Vulcanologia, Sezione di Palermo, Via U. La Malfa 153, Palermo, Italy \\ Received: 4 December 2009 - Revised: 15 October 2010 - Accepted: 17 November 2010 - Published: 10 December 2010
}

\begin{abstract}
In this paper, spectral and detrended fluctuation analyses, as well as time reversibility and magnitude-sign decomposition, have been applied to the 10-year time-series data resulting from geochemical monitoring of gas emissions on the flanks of Mt. Etna, and gases from a $\mathrm{CO}_{2}$ exploitation well located tens of kilometers from the volcano. The analysis of the time series which showed main effects of fractionation between gases due to selective dissolution in aquifers (e.g., the $\mathrm{CO}_{2}$ concentration series), revealed the occurrence of random fluctuations in time, typical of systems where several processes combine linearly. In contrast, the series of He isotopic composition exhibited power-law behavior of the second-order fluctuation statistics, with values of the scaling exponent close to 0.9. When related to the spectral exponent, this value indicates that the isotopic series closely resemble fractal flicker-noise signals having persistent long-range correlations. The isotopic signals also displayed asymmetry under time reversal and long-range correlation of the associated magnitude series, therefore it was statistically proved the presence of nonlinearity. Both long-range correlation and nonlinearity in time series have been generally considered as distinctive features of dynamic systems where numerous processes interact by feedback mechanisms, in accordance with the paradigm of self-organized criticality (SOC). Thus, it is here proposed that the system that generated the isotope series worked under conditions of SOC. Since the fluctuations of the isotope series have been related to magma degassing, the previous results place constraints on the dynamics of such process, and suggest that nonequilibrium conditions must be dominant. It remains unclear whether the signature of SOC is directly due to volatile degassing from magma, or if it derives from the interaction between melt and the stress field, which
\end{abstract}

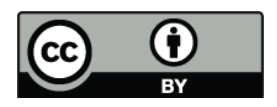

Correspondence to: A. Paonita (a.paonita@pa.ingv.it) certainly influences magma decompression. The strength of scaling appears to increase after 2002 ( $\alpha$ values from 0.8 up to 1.2), focusing on transition of the Etnean system from typical SOC toward conditions of lower criticality. By comparing this transition with those of geophysical observables, it can be suggested that the drop in the rate of magma supply, subsequent to the paroxysms of 2001 and 2002-2003, was the main cause of the scaling change.

\section{Introduction}

Recent studies of Mt. Etna volcano have analyzed the complex behavior of this system by applying modern statistical tools designed for dynamic systems to geophysical data sets obtained by intensive monitoring of the volcano (Centamore et al., 1997; Vinciguerra and Barbano, 2000; Telesca et al., 2002; Vinciguerra, 2002; Currenti et al., 2005a, b; Walter et al., 2005). This approach has revealed previously hidden features of the space and time dynamics of the Mt. Etna system, which suggest it works in conditions of self-organized criticality (SOC); namely, that the system is formed from many interacting components working far from equilibrium, and is characterized by (i) highly nonlinear behavior, (ii) slow driving forces and small perturbations, and (iii) scale invariance of observables in space and time (Sornette and Sornette, 1989; Turcotte, 1992). The first condition means that the effects of individual processes do not simply sum in the system, instead producing chain reactions, and the third condition indicates that the statistics of an observable of the system does not change with the scale of observation (i.e., there are not preferential scales). Scaling has been found in time series of Mt. Etna eruptions (Telesca et al., 2002), which display a fractal distribution and clustering of events. Similarly, power-law distributions (i.e., scale invariance) have

Published by Copernicus Publications on behalf of the European Geosciences Union and the American Geophysical Union. 


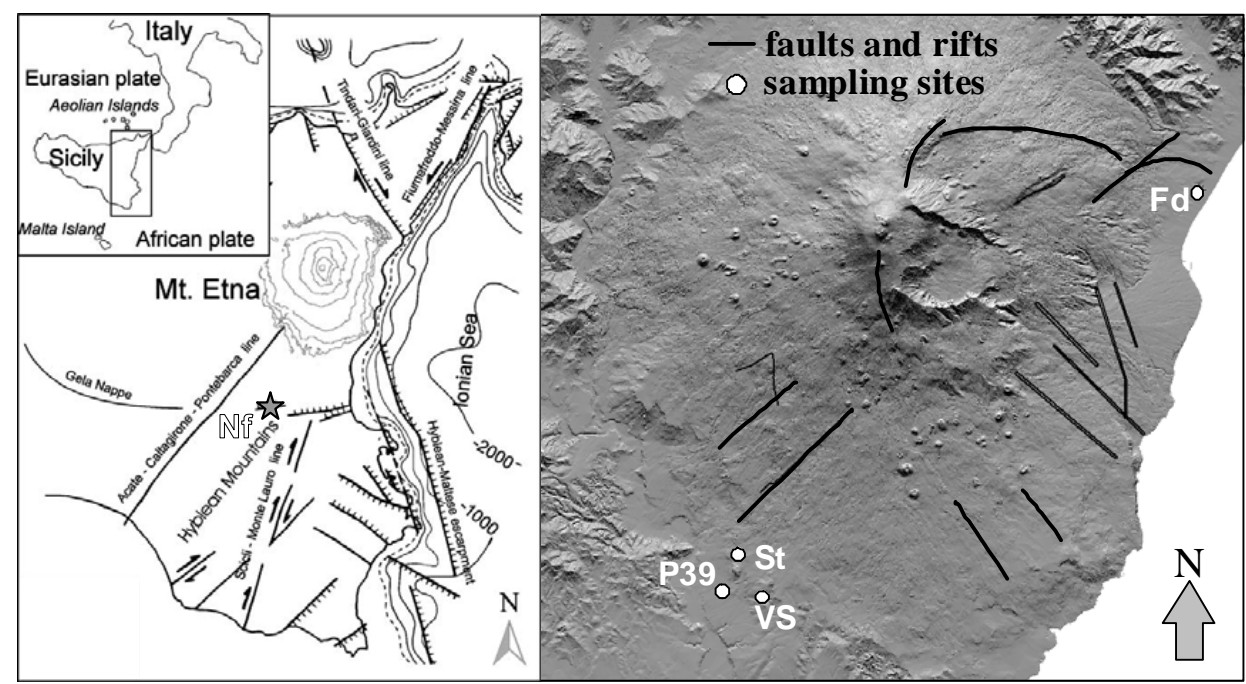

Fig. 1. Sketch map of Eastern Sicily and detail of Mt. Etna, with positioning of main tectonic lineaments and faults (modified from Patanè et al., 2006). Sampled gas discharges are also displayed (see text for details).

been identified by analyzing seismic and geomagnetic signals of Mt. Etna (Currenti et al., 2005a, b). These signals display changes in their time structures, clearly related to the main eruptive episodes, which in turn suggest relationships with magma dynamics whose cause-and-effect mechanisms still need to be clarified. Scale invariance and clustering of seismicity also depends on the main tectonic directions at Mt. Etna (Vinciguerra and Barbano, 2000), accounting for the effects of local and regional stress fields on the volcanic activity (Patanè et al., 2006).

Seismicity and geomagnetic signals are linked to the dynamics of magma (Currenti et al., 2007), whose eruption is the ultimate expression of its ascent. Magma ascent also causes decompression and degassing of volatiles from the silicate melt. Thus, if the above-mentioned observables of Mt. Etna (from geophysical signals to eruptions) exhibit time-scaling behavior, any indicator of magma degassing would also be expected to display scale invariance and an organized temporal structure. Geochemical signals are the best candidate for investigating this, provided that degassingrelated parameters are selected and sufficiently long time series are available. Caracausi et al. (2003a, b) and Rizzo et al. (2006) provide geochemical data in 10-year-long time series from peripheral gas discharges from soils and mud volcanoes of Mt. Etna. The data set comprises chemical and isotope compositions of four gaseous manifestations located on the east and southwest slopes of Mt. Etna, as well as the Naftia gas emissions located on the Hyblean foreland far from the volcano, along the northeast-southwest-trending fault. Caracausi et al. (2003a, b) focused on both the He isotope signals and the concentrations of $\mathrm{He}$ and $\mathrm{CO}_{2}$. They concluded that the observed variations in the He isotope composition are directly linked to magma degassing at depth, while shallower secondary processes modify the original chemical composition of the magmatic gas to various extents. The fractionation of volatiles due to selective dissolution of gas species in shallow aquifers was recognized as the main secondary process affecting the magmatic gases.

In this study, we investigate the scaling (two-point correlation) and nonlinear properties of the geochemical time series from the Mt. Etna peripheral gas discharges. We selected the $\mathrm{He}$ isotope time series obtained at five monitored sites, as well as significant time series of the $\mathrm{He}$ and $\mathrm{CO}_{2}$ concentrations. Each time series consisted of some hundreds of data points collected during 10 years of sampling (since 1996), and they displayed remarkable changes linked to the phases of Mt. Etna activity (Caracausi et al., 2003a, b; Rizzo et al., 2006). We used both isotope and chemical series to highlight differences in the dynamics of magma-related processes with respect to secondary nonmagmatic processes. We show that $\mathrm{He}$ isotope time series (an indicator of magma degassing) exhibit scale invariance, multifractality, long-range correlations and nonlinearity, similar to other geophysical signals. In contrast, the effects of secondary crustal processes appear to be much more random. Finally, our analysis reveals that temporal variations in scaling properties could be related to the activity of the volcano.

\section{The geochemical data set}

Gases discharged from emissions around the periphery of Mt. Etna volcano have been geochemically monitored since 1996 (Caracausi et al., 2003a). Samples were taken from the Vallone Salato mud volcanoes (hereafter called VS) and a soil gas emission near the village of Paternò (hereafter called 
P39) in the southern flank of the volcano (Fig. 1) with a sampling interval of 2 weeks or less. The discharged gases were collected by means of a stainless-steel funnel in the case of mud volcanoes, or through a probe inserted $50 \mathrm{~cm}$ into the emission orifice as regards soil gases. Two-way Pyrex bottles with vacuum valves at both ends were connected to the system and used as sample containers. At the same times, samples were taken from the $\mathrm{CO}_{2}$ exploitation wells of Naftia (hereafter called Nf, see Fig. 1), located on the Hyblean foreland $40 \mathrm{~km}$ from the volcano, along the northeast-southwesttrending fault. In this case, the two-way sample containers were directly connected to the head of the well. In all the samples, the He isotope ratio was always measured, while the entire chemical composition was regularly analyzed from the year 2000 onwards. Two other gas discharges were added to the monitoring program in 2001: (i) the mud volcanoes of Salinelle di Stadio (hereafter called St) at Paternò village, and (ii) the bubbling gases of the Fondachello water channel (hereafter called Fd) along the coast east of the volcano (Fig. 1) (Caracausi et al., 2003b; Rizzo et al., 2006). Since then, all five sites have usually been sampled at a rate of once every 2 weeks, with it being increased to one or even two samples per week during periods of unrest of the volcano. As a result, we have (i) 10-year time-series data of He isotope ratios from sites VS, P39, and Nf, with each series consisting of about 350 data points; (ii) shorter isotope series from sites Fd and St (each comprising $\sim 200$ data points); and (iii) time series of chemical concentrations for every measured species at each of the five sites, comprising 200-250 points. These series are, at our knowledge, the longest and complete collections of $\mathrm{He}$ isotopic data from an active volcano. When recalling that an operator is required to collect these type of samples, it is easy to understand the huge field work required to build this dataset. Despite the big efforts to maintain regular sampling frequencies, the changeable field and weather conditions, as well as any logistical questions in a ten-year monitoring, made impossible to achieve regularly sampled data stricto sensu. Shift by a few days are thus present, as well as some gap of data.

The He isotope composition of the gas discharges (6.7 \pm $0.9 R_{\mathrm{a}}, R_{\mathrm{a}}$ being the isotope ratio in the atmosphere) suggest a magmatic genesis, since it matches that of He trapped in olivine phenocrysts of Etnean lavas $\left(6.7 \pm 0.4 R_{\mathrm{a}}\right.$; Marty et al., 1994). The very high $\mathrm{He} / \mathrm{Ne}$ ratios of the emissions rule out any appreciable contribution of $\mathrm{He}$ from the atmosphere. With this in mind, Caracausi et al. (2003a) focused on large temporal variations in the $\mathrm{He}$ isotopes. By using chemical balances between the magmatic fluid and the local crustal endmember, as recognized in several $\mathrm{CH}_{4}$ exploitation wells in the area, they showed that the isotope fluctuations were not due to variable contributions of crustal He. Those authors also observed that the large changes occurred simultaneously at all sites, even those $60-\mathrm{km}$ distant, and a few months prior to the main eruptive phases of Mt. Etna (2001, 2002-2003, and 2006). All these features allowed them to link the variations to magmatic processes occurring inside the Etnean plumbing system. The isotope changes were attributed to episodes of rapid volatiles exsolution from magma due to melt ascent and decompression during the refilling of an extensive plumbing system.

Caracausi et al. (2003b) identified more complex variations in the chemical composition of the emissions. Coupled to the effects of magma degassing, those authors found evidence of chemical dissolution of the magmatic fluids into shallow hydrothermal aquifers. The gas species exhibit, in fact, different solubilities in water: while $\mathrm{CO}_{2}$ dissolves in the aqueous solution, noble gases, $\mathrm{N}_{2}$, and $\mathrm{CH}_{4}$ are selectively enriched in the gas phase at chemical equilibrium with liquid water. Modeling such secondary processes allowed Caracausi et al. (2003b) to filter out the changes of gas composition due to the selective dissolution into aquifers and to obtain the composition of the pristine magmatic gas phase. The dissolution-driven effects largely differed among the sites, ranging from very modest dissolution at $\mathrm{Nf}$, up to the almost total $\mathrm{CO}_{2}$ removal at $\mathrm{Fd}$, where a $\mathrm{CH}_{4}$-dominated gas phase is discharged. Moreover, in the case of site VS, the degree of gas dissolution also changed in space and time, causing large variations in the chemical composition. After filtering out such secondary effects, Caracausi et al. (2003b) identified temporal variations in both $\mathrm{He} / \mathrm{CO}_{2}$ and $\mathrm{He} / \mathrm{Ne}$ ratios, consistent with processes of chemical fractionation during magmatic degassing. Based on the solubilities of these volatiles in silicate melts, Caracausi et al. (2003b) computed the decompression of the magma bodies involved in the episodes of refilling, and assessed the depths of the two major magmatic reservoirs. It is noteworthy that the estimated depths were similar for all the sites except for $\mathrm{Nf}$.

\section{Statistical methods}

As stated in Sect. 2, our geochemical series (hereafter GS) must be regarded as unevenly sampled time series from a strictly statistical point of view, even if they contain a largely dominant sampling frequency. Moreover, similar to many types of physical, biological, financial and Earth Science data (Kantz and Schreiber, 2004; Lovejoy and Schertzer, 2007a, b), these series are noisy and strongly nonstationary signals, in that they display intrinsic fluctuations at all scales together with embedded local trends (Caracausi et al., 2003a, b; Rizzo et al., 2006; see also Fig. 12a). To test the occurrence of long-range correlation in such signals, other statistical methods than autocorrelation and power spectrum analysis have been more conveniently applied (Kantelhardt et al., 2001), although they can hardly work out in the case of unevenly sampled series (Schmitz and Schreiber, 1999). Taking into account the above raised issues, we follow two approaches:

1. we try to qualitatively reveal the occurrence of longrange correlation and nonlinearity in our GS by 
applying specific statistical tools for unevenly sampled time series;

2. we make an effort for quantifying the scaling and nonlinear properties of the GS by using statistical methods which are particularly suitable for very fluctuating signals. Because such methods work with evenly sampled time series, they require an interpolation of the GS.

In both the approaches, we applied the surrogate series method (Theiler et al., 1992), with the aim to assess the statistical confidence of our results and the effects of the interpolation procedures. In the following, we detail about the applied statistical approaches.

\subsection{Analysis of unevenly sampled time series}

We firstly tried to put into evidence, from a qualitative point of view, long-range correlation and nonlinearity in unevenly sampled GS. Long-range correlations were investigated by calculating the power spectrum of GS and searching for any power law distribution of their spectral frequencies, in accordance with the relation:

$S(\omega) \sim \omega^{-\beta}$

where $S(\omega)$ is the power spectrum of the GS, $\omega$ is the spectral frequency and $\beta$ is the spectral exponent of power law. Because the well-known Fourier transform cannot be calculated for unevenly sampled series, we used the Lomb periodogram (Lomb, 1976) to achieve the power spectrum.

Afterward, we applied the test of Time Reversibility (hereafter TR) to the GS, which allows to put qualitatively into evidence any nonlinearity in unevenly sampled time series (Schmitz and Schreiber, 1999). The TR test analyzes the first-differences of series raised to third power, therefore is a measure of asymmetry of the series under time reversal (Subba Rao and Gabr, 1984; Dikes et al., 1995). Since linear stochastic processes are fully characterized by their power spectrum, which does not contain information on the direction of time, any time asymmetry of series reveals the presence of nonlinearity. For a time series of data $x(i)$ sampled at times $t(i)$, with $i=1,2, \ldots N$, we define TR as (Schmitz and Schreiber, 1999):

$\gamma=\frac{1}{\left(\sigma^{2}\right)^{3 / 2}(N-1)} \sum_{i=2}^{N}\left(\frac{x_{i}-x_{i-1}}{t_{i}-t_{i-1}}\right)^{3}$

where $\gamma$ is the measure of the TR, and $\sigma$ is the standard deviation of the time series. Conceptually, Eq. (2) computes the mean of the slopes at the third power. For linear stochastic processes, which exhibit the same statistic properties under time reversal, we can expect to calculate $\gamma=0$ in Eq. (2). In contrast, the achievement of $\gamma$ values other than zero indicates that the time series is somewhat nonlinear.

The TR test has been very frequently used for analyzing several types of time series (see Schmidtz and Schreiber,
1999; Gliozzi et al., 2002; Porta et al., 2006). The statistical properties of the handled series such as probability density function (hereafter PDF) and autocorrelation, have been able to affect the results of the test (Schreiber and Schmitz, 1997; Schmitz and Schreiber, 1999; Gliozzi et al., 2002), therefore the reliability of the method must be estimated by numerical experiments based on a suitable null hypothesis (see Sect. 3.3.2). In numerical studies, TR has achieved highquality performances, sometimes better than other nonlinearity tests (i.e. correlation sum and high-order autocorrelation; Schreiber and Schmitz, 1997). The occurrence of time asymmetry has proved to be a sufficient condition to declare that the time series shows nonlinear properties. On the other hand, it cannot be regarded as a necessary condition, given that the test was not able to reject the null hypothesis of linearity for some types of noisy nonlinear signals (Schreiber and Schmitz, 1997).

\subsection{Time series interpolation, and quantification of scaling and nonlinearity}

After the qualitative recognition, our main aim was to quantify the long-range correlation and nonlinear properties of the GS by coupling the detrended fluctuation analysis (Peng et al., 1995) and the magnitude-sign decomposition (Ashkenazy et al., 2001, 2003), which are known to work well for very fluctuating signals. Because these methods are conveniently applied to regularly sampled time series, we were forced to interpolate our GS signals. We used the two simplest methods of interpolation: (i) a step-like scheme, by dividing the time interval between samples $x_{i}$ and $x_{i+1}$ into two equal parts, and assigning the value $x_{i}$ to the $x_{i}$-edged half and the value $x_{i+1}$ to the $x_{i+1}$-edged half; and (ii) a piecewise first-order polynomial scheme, consisting of joining adjacent samples with straight lines (hereafter referred to as linear, in contrast with the step-like interpolation). We then sampled the interpolated series with a constant time step so as to achieve evenly spaced time series, hereafter called regular GS (RGS), as distinct from the GS. On the basis of our preferential field sampling at intervals of 1 and 2 weeks, the sampling frequencies of the new RGS were chosen to match these two main frequencies (that is 0.07 and 0.14 day $^{-1}$ ). The RGS allow that both detrended fluctuation analysis and magnitude-sign decomposition can be performed.

\subsubsection{Detrended fluctuation analysis}

The Detrended Fluctuation Analysis (DFA) was specifically developed to analyze long-range correlation in noisy and nonstationary signals with embedded trends by Peng et al. (1995), and it has been successfully employed to identify scaling in hundreds of time-series data from totally different research fields (see Goldberger et al., 2000, for a review). In DFA, the time series $x(i)$, with $i=1,2, \ldots, N$, is first meansubtracted and then integrated: 
$y_{k}=\sum_{i=1}^{k}\left(x_{i}-\bar{x}\right)$

where $x_{i}$ is the $i$-th element and $\bar{x}$ is the mean of the time series. The $y(k)$ series (called the profile function) is divided into $M=\operatorname{Int}(N / n)$ time windows having size $n$, indexed by $v=1,2, \ldots, M$. Since the length of $y(k)$ is normally not a multiple of $n$ and a short final segment of $y(k)$ is not analyzed, the profile is also calculated starting from the other end, thereby producing $2 M$ windows. Each window $v$ is fitted by an $m$-th order polynomial $p^{m}$ with $m<n$, so that $m$ defines the order of the DFA. Finally, the fluctuation function is computed as:

$F(n)=\sqrt{\frac{1}{2 M} \sum_{v=1}^{2 M} \frac{1}{n} \sum_{k=1}^{n}\left[y_{(v-1) n+k}-p_{(v-1) n+k}^{m}\right]^{2}}$

When $x(i)$ displays long-range correlation, $F(n)$ follows the power-law relation:

$F(n) \propto n^{\alpha}$

where $\alpha$ is the scaling exponent, which is computed as the slope of the best-fit straight line in a plot of $\log [F(n)]$ vs. $\log (n)$. Equation (4) becomes highly unstable on long timescales (high $n$ values) due to the few windows considered, so that it is usually interrupted when $n>N / 4$ (Kantz and Schreiber, 2004). In addition, it is known that the $F(n)$ values are underestimated relative to a pure power-law prediction on a short timescale $(n<10$; Hu et al., 2001; Kantelhardt et al., 2001).

In the case of stationary Gaussian stochastic series, the scaling exponent $\alpha$ can be analytically related to the autocorrelation exponent $\gamma$ (see Taqqu et al., 1995). Taking into account that $\gamma$ and $\beta$ are linked through the Wiener-Khinchin theorem, it follows that a relationship also exists between the spectral and the scaling exponents:

$\beta=2 \alpha-1$

Also, the exponent $\alpha$ can be related to the well-known Hurst exponent $\mathrm{H}$. For stationary series such as fractional Gaussian noises (having $-1<\beta<1$ ), the profile function $y_{k}$ of Eq. (3) will be a fractional Brownian motion (Brownian motion is in effect the integral of Gaussian noise) and the scaling exponent will be $0<\alpha<1$, as computed by Eq. (6). In this case, $\alpha$ will be identical to $\mathrm{H}$ (Taqqu et al., 1995), which in fact takes values between 0 and 1 . For non-stationary signals, such as fractional Brownian motion with $1<\beta<3$, the profile $y_{k}$ will be the integral of a fractional Brownian signal and will display scaling exponents in the range $1<\alpha<2$ (by Eq. 6). It follows that the exponent $\mathrm{H}$ of the fractional Brownian signal will be equal to $\alpha-1$.

By means of Eq. (6), we can also define the values of $\alpha$ exponent to be expected for Gaussian stochastic signals having known spectral and correlation properties. In fact, we derive that $\alpha=0.5$ for uncorrelated time series, typical of ran- dom white-noise processes having $\beta=0$. Long-range correlated data will give higher or lower values: $\alpha<0.5$ indicates anticorrelated series (where a large value is more likely to be followed by a small value); $\alpha>0.5$ indicates correlated (persistent) time series; $\alpha=1$ is typical of dynamic systems in a self-organized critical state (producing flicker noise, also called $1 / f$ or pink noise); and $\alpha=1.5$ finally suggests integrated white-noise series, typical of Brownian-like processes.

As concerns non-Gaussian stochastic series, several studies have been aimed to evaluate possible effects of probability distributions other than Gaussian on results of both DFA and other correlation estimators (see Barunik and Kristoufek, 2010, for a short review). Although the conclusions of these works do not always agree about some questions, here we recall the main achieved results which can be relevant to our work. The first one is that the expected scaling exponent of uncorrelated time series is 0.5 , regardless the PDF of the signal. Kantelhardt et al. (2002) showed analytically this result for long-tailed distributions too. Secondly, all the correlation estimators, including DFA, suitably work in the case of Gaussian and near-Gaussian distributions, while they deteriorate their performances with increasing heavy tails of PDF (Barunik and Kristoufek, 2010). Therefore, the confidence level of the DFA test has to be specifically evaluated depending on the properties of handled series (Sect. 3.3.1).

\subsubsection{DFA in short time series}

Time series comprising less than $10^{3}$ points, such as the GS in this work, may adversely affect the reliability of the estimations of $F(n)$ and $\alpha$, although some studies suggest that DFA is poorly influenced by such finite-time scale effects (Cernelc et al., 2002; Colorado and Carpena, 2005). We chose to improve the confidence of the estimated exponents by using the technique of the phase randomized surrogate (PRS; Govindan et al., 2007), specifically addressed to minimize this question. It involves creating several copies of the series by preserving the correlations of the original series. These surrogate series are generated by firstly computing the Fourier transform $F(\omega)$ of $x(i)$ after subtraction of the mean:

$F(\omega)=|u(\omega)| e^{i \Phi(\omega)}$

where $|u(\omega)|$ and $\Phi(\omega)$ represent the magnitude and phase angle of the transform, respectively. The phase is substituted by using the phase of an equal-length series of independent and identically distributed (i.i.d.) Gaussian random numbers. An inverse Fourier transform is then computed to obtain the phase-randomized series. Since:

$S(\omega)=F(\omega)^{2}=|u(\omega)|^{2}$

and the two series (original and surrogate) have the same $u(\omega)$, they also display the same power spectrum. It follows from Eq. (6) that they must theoretically have the same value of $\alpha$. Therefore, the $F(n)$ function is computed for 
both the original and surrogate series, and the result is averaged among all the achieved values to reduce the statistical variations in $F(n)$.

Following Govindan et al. (2007), a number of 39 surrogate series must be used to achieve a $95 \%$ significance level of the estimated exponent when using the PRS technique. Since the surrogate series exhibit slightly different variances, they are normalized by their standard deviation before applying DFA. Both original and surrogate series display the above-mentioned underestimation of $F(n)$ on short timescales (see Sect. 3.3), which reduces the range of timescales that can be used for best fitting and $\alpha$ estimation. For short data sets it is hence useful to apply the correction proposed by Kantelhardt et al. (2001), which should eliminate or at least reduce such underestimation at small $n$ values. Accordingly, $F(n)$ is multiplied by a correction function $K(n)$ that, by construction, has negligible effects at large timescales, whereas it gets growing weight as soon as $n$ becomes small.

\subsubsection{Magnitude and sign decomposition}

Long-range correlated time series can exhibit single or multiple Hurst exponents, and hence are named mono- or multifractal, respectively. Whereas monofractal series display linearly dependent exponents of the different moments, multifractal ones are nonlinear. It can be shown that mono- and multifractal series having similar long-range correlation will also display the same power-law distribution of Fourier amplitudes. In contrast, the monofractal series will have a totally uncorrelated phase spectrum, whereas the phase spectrum of the multifractal series will be still correlated. To shuffle the Fourier phases of the multifractal series therefore converts the multifractal series into a monofractal one, destroying the nonlinearity. On this basis, Ashkenazy et al. $(2001,2003)$ proposed a practical method to distinguish linear from nonlinear signals. The method involves first computing the increment series from the original time series $x_{i}$ :

$\Delta x_{i}=x_{i+1}-x_{i}$

Opposite to an integration (see Eq. 3), Eq. (9) results in the correlations of the increment series being characterized by an exponent that is about 1 less than the original series. Thereafter, the magnitude series $\left|\Delta x_{i}\right|$ and the sign $\operatorname{series} \operatorname{sgn}\left(\Delta x_{i}\right)$ are computed from increments, so that:

$\Delta x_{i}=\operatorname{sgn}\left(\Delta x_{i}\right)\left|\Delta x_{i}\right|$

Based on numerical experiments, Ashkenazy et al. (2003) showed that the sign series preserves the linear correlations of the increment series (so producing $\alpha_{\text {incr. }} \cong \alpha_{\text {orig. }}-1$ ). Instead, the correlation of the magnitude series provides information about the nonlinearity of the original series $x_{i}$. This means that if the original series is positively correlated (e.g., $\left.\alpha_{\text {orig. }}=1\right)$ and nonlinear, the $\alpha$ value of the magnitude series is also suggestive of a long-range correlation (namely, $\alpha_{\text {magnitude }}=1$ ), whilst both the sign and increment series will be anticorrelated. Randomizing the Fourier phases of the original series so as to destroy its nonlinearity will not affect the correlations of both the increment and sign series, but the magnitude series will appear to be totally uncorrelated $(\alpha=0.5)$.

The same as in the other statistics of this work, the magnitude test can be also affected by both autocorrelation property and PDF of the handled time series, therefore the null hypothesis and the confidence level of the test have to be evaluated by numerical experiments (Sect. 3.3.1). Recently, Nagarajan (2007) achieved suitable results in rejecting null hypothesis of linearity when using magnitude series of Gaussian and near-Gaussian signals, coupled to PRS method for surrogates. In the case of strongly non-Gaussian signals, magnitude did not work suitably when coupled to surrogate techniques which preserve the non-Gaussianity of PDF (Nagarajan, 2007).

\subsection{Reliability of the statistics: the method of surrogate series}

The method of surrogate series (Theiler et al., 1992) allows to estimate reliability and error bars of a statistical test when we do not know its probability distribution and cannot analytically calculate its variance. Practically, one creates several surrogate series having chosen PDF and correlation properties, and applies the selected statistic test on these surrogates. The comparison of the achieved results with the expected ones constrains the reliability and uncertainty of the test. Here we used the surrogate series method on: (i) DFA and magnitude-sign decomposition studies of the interpolated geochemical series; (ii) the TR tests for unevenly sampled geochemical series.

\subsubsection{DFA and magnitude-sign decomposition on interpolated series}

The surrogate series with chosen correlation features were generated by using the Fourier filtering method described in Chen et al. (2002). In detail, coupling Eqs. (1) and (8) yields:

$u(\omega)=F(\omega) \sim \omega^{-\beta / 2}$

In the case of a Gaussian white noise, the latter one has Fourier transform $F_{G}(\omega)$ with spectral exponent $\beta$ equal to 0 (the subscript "G" refers to Gaussian white series). Fourier coefficients having a known power-law distribution can be obtained using:

$u(\omega)=F_{G}(\omega) \omega^{-\beta / 2}$

where $\beta$ is the chosen spectral exponent. We therefore generated a Gaussian white series with similar variance to original data, we calculated the Fourier coefficients, and used them in Eq. (12) with the selected $\beta$ value (namely, the required correlation property). An inverse Fourier transform performed 


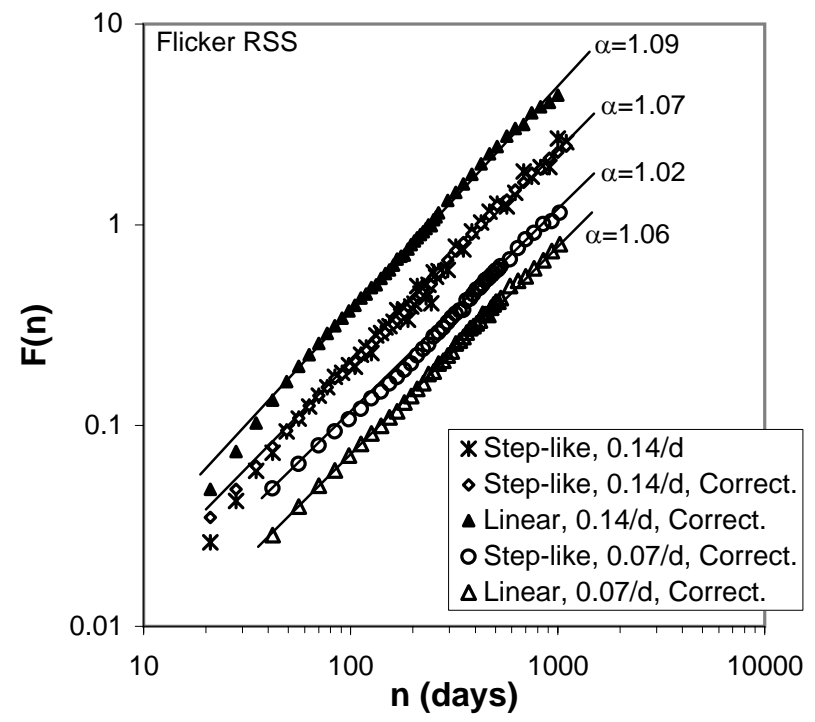

Fig. 2. Bilogarithm plot of the fluctuation function $F(n)$ vs. time scale $n$, for a RSS having flicker-noise correlation properties (theoretical $\alpha=1.03$ ). The DFA results are displayed for the series interpolated by both step-like and linear method, and resampled by both 0.07 day $^{-1}$ and 0.14 day $^{-1}$ frequencies. The label "correct." refers to DFA coupled to PRS and small- $n$ correction techniques (see text). As the calculated $F(n)$ functions almost overlap, they have been multiplied by an arbitrary factor so as to be shifted for clarity. This procedure was repeated in the following figures whenever required.

thereafter on the Fourier coefficients coming from Eq. (12) yielded stationary data having the chosen correlation property [hereafter named the simulated series (SS)]. Equation (6) also gave the expected value of scaling exponent $\alpha$.

It should be noticed that this technique produces correlated series having expected Gaussian distribution. We already discussed on the fact that DFA exhibits very good performance when we work with Gaussian to near Gaussian series, while progressively reducing its quality with increasing heavy tails of PDF (Barunik and Kristoufek, 2010), therefore surrogates should have similar PDF than the original series. By using simple histograms, it can be showed that the GS essentially are Gaussian to near-Gaussian distributed signals. We took care to compare the PDF of GS to that of the surrogate series, and we verified a good match of the distributions. As a result, the effects of PDF on DFA were considered to be minor.

We applied the interpolation procedure to the SS with a known $\alpha$ value and length equal to that of the GS, and calculated a new series (hereafter called regular SS (RSS), by analogy with the RGS) by sampling the interpolated SS at the same frequency as that used for the RGS. Then, we applied DFA to the RSS, and we compared the achieved results with those expected.

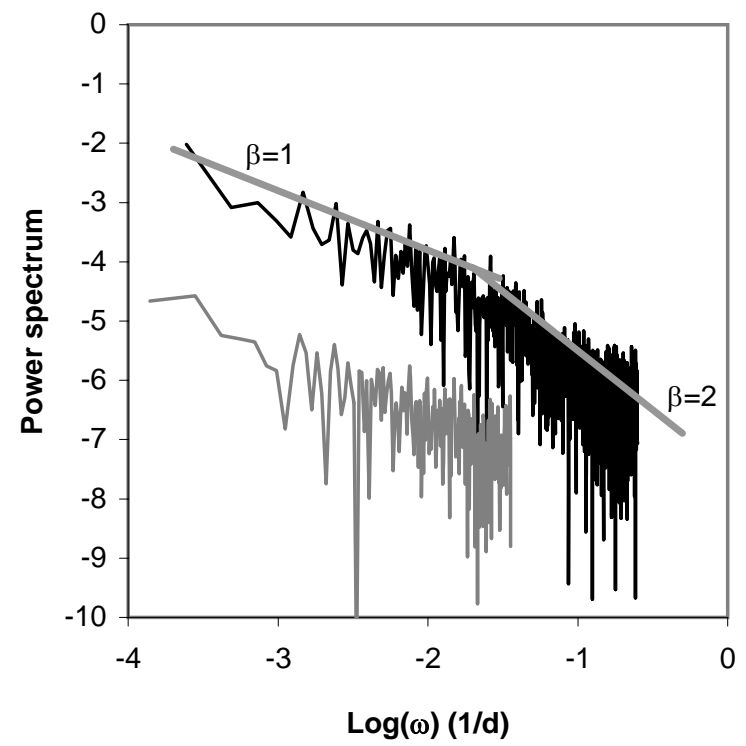

Fig. 3. Power spectrum of a flicker RSS, with step-like interpolation and resampled by $0.07 \mathrm{~d}^{-1}$ (grey) and 0.14 day $^{-1}$ (black). The two curves have been shifted by 2 logarithm units for clarity. The different spectral exponent of the high- and low-frequency branches is displayed.

Figure 2 shows a $\log [F(n)]$ vs. $\log (n)$ plot for a flicker RSS. It is clear the advantage of using the techniques for improving DFA of short time series (i.e. the coupled PRS and small- $n$ correction techniques, see Sect. 3.2.2), in that they greatly reduce the statistical variability of the estimated $F(n)$ values. We therefore used these two techniques as a rule, nonetheless their application does not change basically the meaning of our analyses.

In RSS with weekly sampling $\left(0.14\right.$ day $\left.^{-1}\right)$, all $F(n)$ functions show underestimations at small scales $(n<49$ days, or $n<7$ points), suggesting that the small- $n$ correction method only partially solves the problem (Fig. 2). The deflection does not appear in the corrected DFA of uninterpolated SS, indicating that it is caused by the interpolation procedure. Indeed, interpolation schemes are known to affect estimations of power spectra, since they reduce the high-frequency components. In fact, the step-like scheme causes a power-law form of the high-frequency portion of spectrum with $\beta=2$, whereas linear interpolation gives $\beta=4$ (Newman and Eble, 1999). In Fig. 3, this purely mathematical effect is evident at high frequencies, with a crossover from high- to lowfrequency behavior occurring around $0.02 \mathrm{day}^{-1}$ (timescales of about 50 days). Since the power spectrum exponent $\beta$ and scaling exponent $\alpha$ are linked (Eq. 6), higher $\beta$ values for timescales shorter than 50 days imply higher $\alpha$ (with values of 1.5 to 2.5 , for step-like to linear interpolations, respectively); this explains the above-mentioned deflection. This problem constrained us to exclude the values of $F(n)$ for $n<49$ days when fitting the straight line to estimate $\alpha$. In RSS with a sampling frequency of 0.07 day $^{-1}, F(n)$ cannot 


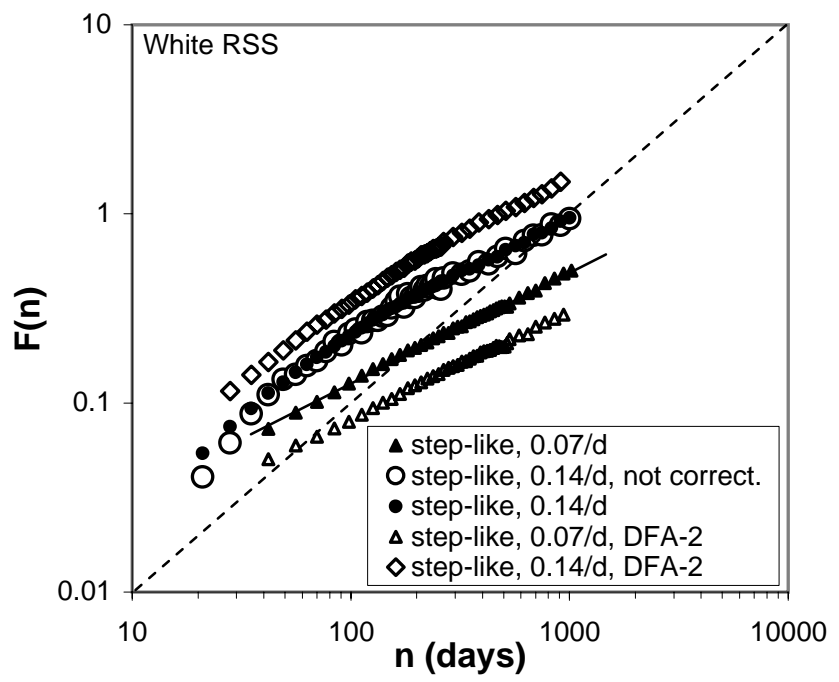

Fig. 4. Fluctuation function $F(n)$ vs. time scale $n$, for a RSS having white noise correlation (theoretical $\alpha=0.55$ ). A straight line having slope $\alpha=1$ has been plotted for comparison (dotted). DFA was always coupled to PRS and small- $n$ correction techniques, except for calculation of the data group labeled "not correct.". Resampling frequencies were both $0.14 \mathrm{day}^{-1}$ and $0.07 \mathrm{day}^{-1}$. The label "DFA-2" indicates second-order DFA analysis.

be computed below the scale of 42 days (namely, $n<3$ points), and hence the small-scale deflection is much less evident (Figs. 2 and 3).

Similar results were obtained by applying DFA to whitenoise RSS (Fig. 4), although the small-scale deflection was more marked due to the lesser slope of the $\log [F(n)]$ function for white noise. Indeed, it was already evident for a timescale shorter than 100-120 days when the 0.14-day ${ }^{-1}$ sampling frequency was applied. At a sampling frequency of 0.07 day $^{-1}$, short timescales were only slightly affected by underestimation and thus the $\alpha$ exponent of uncorrelated signals could be still suitably estimated by linear best fitting of the $\log [F(n)]$ function with $n \geq 56$ days (or $n \geq 4$ points). Finally, higher-order DFAs did not change the overall results, but they did cause appreciable interpolation-related underestimation of $F(n)$ on timescales of longer than 100 days (Fig. 4).

With the aim of quantitatively assessing the uncertainties in $\alpha$ estimation, we generated a large number of SS with expected correlations ranging from white to Brownian noise by the surrogate method, and we converted them into RSS by the interpolation and resampling procedure. When applying DFA, we estimated the scaling exponent by fitting the linear portion of the $\log [F(n)]$ function ( $n \geq 49$ or $\geq 56$ days depending on the selected sampling frequency). For frequencies of both 0.14 and 0.07 day $^{-1}$, the comparison between expected and estimated values revealed that the exponent was slightly overestimated for white-noise uncorrelated signals, very similar for flicker noise, and slightly underestimated for
Brownian noise (Fig. 5). Fitting the data distribution in Fig. 5 with a quadratic polynomial curve allowed the expected $\alpha$ value to be calculated from the estimated one, giving standard errors of $0.06-0.08 \alpha$ units.

Surrogate series tests were finally performed to quantify the reliability of the estimated scaling exponent of the magnitude series. In so doing, we were encouraged by the achieved positive results for near-Gaussian signals similar to our GS, already mentioned in Sect. 3.2.3 (Nagarajan, 2007). We focused on evaluating the significance of $\alpha$ values higher than 0.5 , which should be indicative of nonlinearity in the time series. We generated SS with known correlations and similar distribution to that of the GS (see above), and since such series were monofractal, the resulting magnitude series were uncorrelated ( $\alpha \cong 0.5$ ). We then converted the SS into RSS by the interpolation and resampling procedure, computed the magnitude series, and finally applied DFA to the magnitude RSS, predicting that $\alpha \cong 0.5$. As a result, we found that both step-like and linear interpolated RSS with a $0.14-$ day $^{-1}$ sampling frequency displayed some degree of correlation. The assessed average exponents were in fact 0.65 and 0.72 , respectively, with uncertainties of about $0.05 \alpha$ units (Fig. 6). Randomizing the phase spectra of these RSS and again performing DFA yielded the expected $\alpha \cong 0.5$ (Fig. 6). Several RSS with step-like interpolation and a 0.14 -day ${ }^{-1}$ sampling frequency also displayed deviations from a strictly linear behavior in a plot of $\log [F(n)]$ vs. $\log (n)$, giving an estimated $\alpha$ with poor reliability (Fig. 6). As shown in the following, this problem also emerged in RGS resampled at this frequency, and thus we did not take into account the related magnitude exponents. In contrast, using a $0.07-$ day $^{-1}$ sampling frequency yielded plots of $\log [F(n)]$ vs. $\log (n)$ of good quality (Fig. 6). Their average exponents were 0.58 and 0.65 for step-like- and linear-interpolated RSS, respectively (Fig. 7). These values differ little from the expected $\alpha=0.5$, and we took into account such a difference when evaluating the nonlinearity of RGS.

\subsubsection{Reliability of TR tests}

For creating surrogates of unevenly sampled series to be used in TR test, we followed the Constrained Randomization method as described in Schmitz and Schreiber (1999). Accordingly, the power spectrum of the real time series is calculated by using the Lomb periodogram, that provides the constraints for creating the surrogates. Starting from a randomized distribution of the original data, they are annealed until their Lomb periodogram closely resembles that of the original data. The method is based on defining a cost function which strictly depends on the difference between the Lomb periodogram of surrogate data and that of the original series. This function is then minimized by simulated annealing: the surrogate series is modified by exchanging two points chosen at random, and the modification is accepted if it yields a lower value of the cost function. The generated series can 

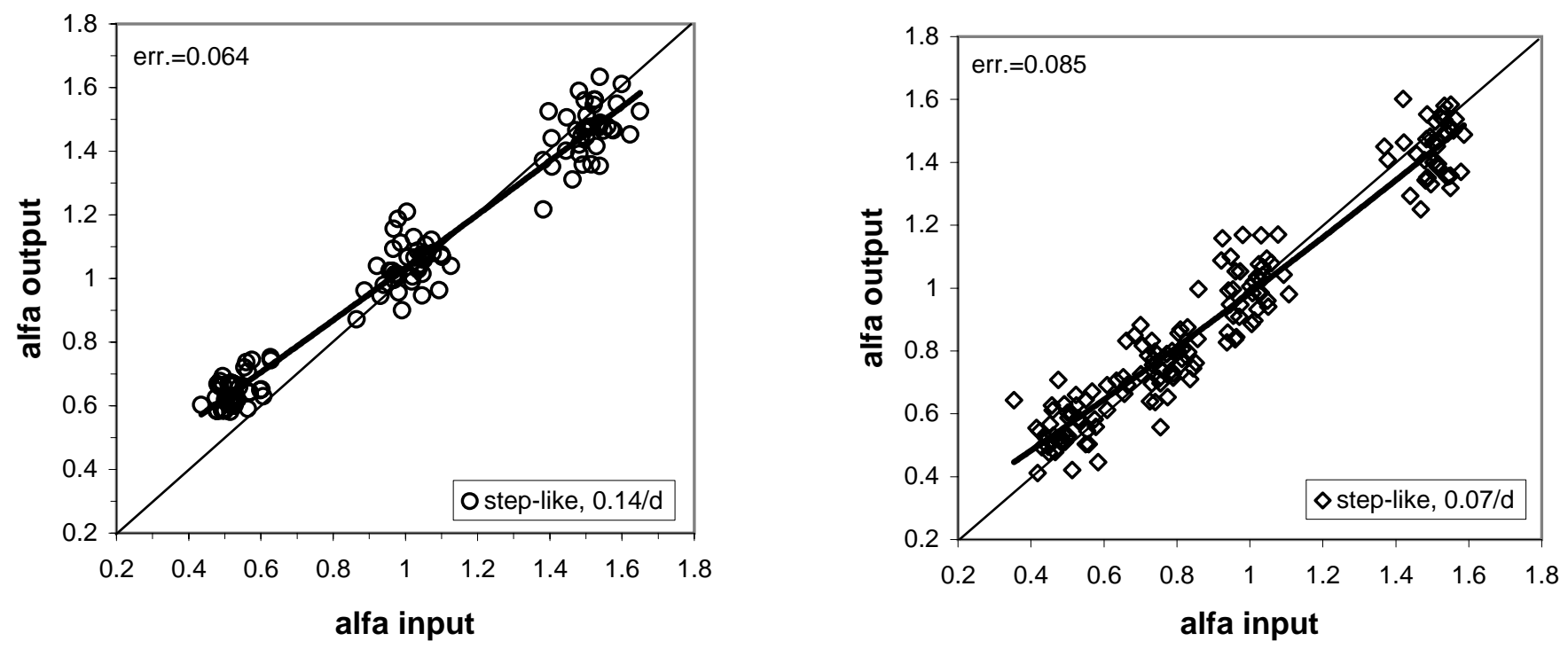

Fig. 5. DFA-calculated vs. expected $\alpha$ exponents for RSS. All the simulated series having known $\alpha$ exponent were step-like interpolated, resampled by the selected frequency $\left(0.14 \mathrm{day}^{-1}\right.$ and $0.07 \mathrm{day}^{-1}$, left and right picture, respectively) and managed by DFA in order to assess the $\alpha$ values. Standard errors of the polynomial fits are also given.

be accepted as surrogate when the cost function becomes smaller than a selected tolerance. The similar Lomb periodogram of surrogate and original series and the annealing procedure guarantee for keeping similar long-range correlation and probability distribution, respectively (namely all the linear properties). In contrast, the surrogate series have lost the nonlinearity of the original series, due to the initial step of randomization. As a consequence, the technique implies that the null hypothesis is a linear stochastic process, regardless its PDF.

Depending on the selected tolerance to accept surrogates, as well as the length of series, the calculation can be very time-consuming. The TISEAN code (Hegger et al., 1999) was employed with this goal. Starting from a GS, we calculated 20 surrogate series having the same correlation properties (namely, very similar $\beta$ value), in order to guarantee a 90\% level of significance for the test (Schmitz and Schreiber, 1999). After, we estimated the parameter $\gamma$ for each surrogate series by using Eq. (2). The obtained values move in the range $-0.05<\gamma<0.05$, meaning that $\gamma$ values of the realdata time series within this range do not allow to exclude linearity, while higher or lower values imply the occurrence of nonlinear properties.

To conclude this section on the reliability of the applied statistics, it is useful to shortly recap the above-discussed effects of the PDFs of time series on the applied techniques of surrogates. The occurrence of non-Gaussianity of PDF can indeed affect the estimations of both correlation and nonlinearity of time series, hence the chosen surrogate method should preserve the PDF of original series. In evaluating long-range correlation by DFA, the applied Fourier filtering technique in Sect. 3.3.1 can in principle modify the PDF of

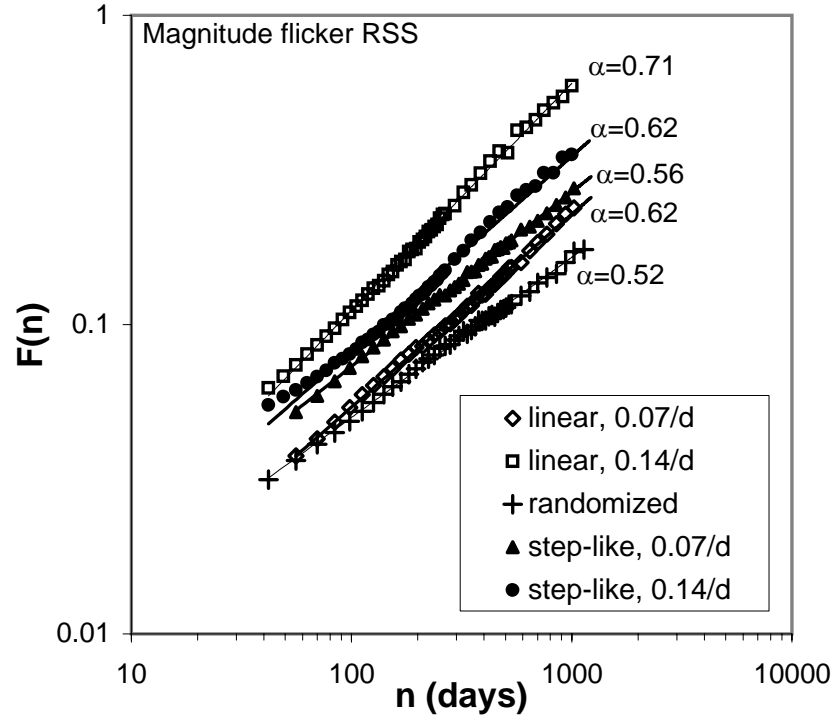

Fig. 6. Log-log plot of the fluctuation function $F(n)$ vs. time scale $n$, for a magnitude RSS. The DFA results are displayed for the series interpolated by both step-like and linear method, and resampled by both $0.07 \mathrm{day}^{-1}$ and $0.14 \mathrm{day}^{-1}$ frequencies. The label "randomized" refers to DFA of the magnitude RSS after that the RSS phase spectrum was randomized (see text).

the series to a Gaussian, nevertheless the quasi-gaussianity of our GS ensured very good match between original and surrogates. For similar reasons, the coupling of magnitude method and Fourier filtering technique provided suitable results in rejecting null hypothesis of linearity when performing nonlinear estimations. On the other hand, in 

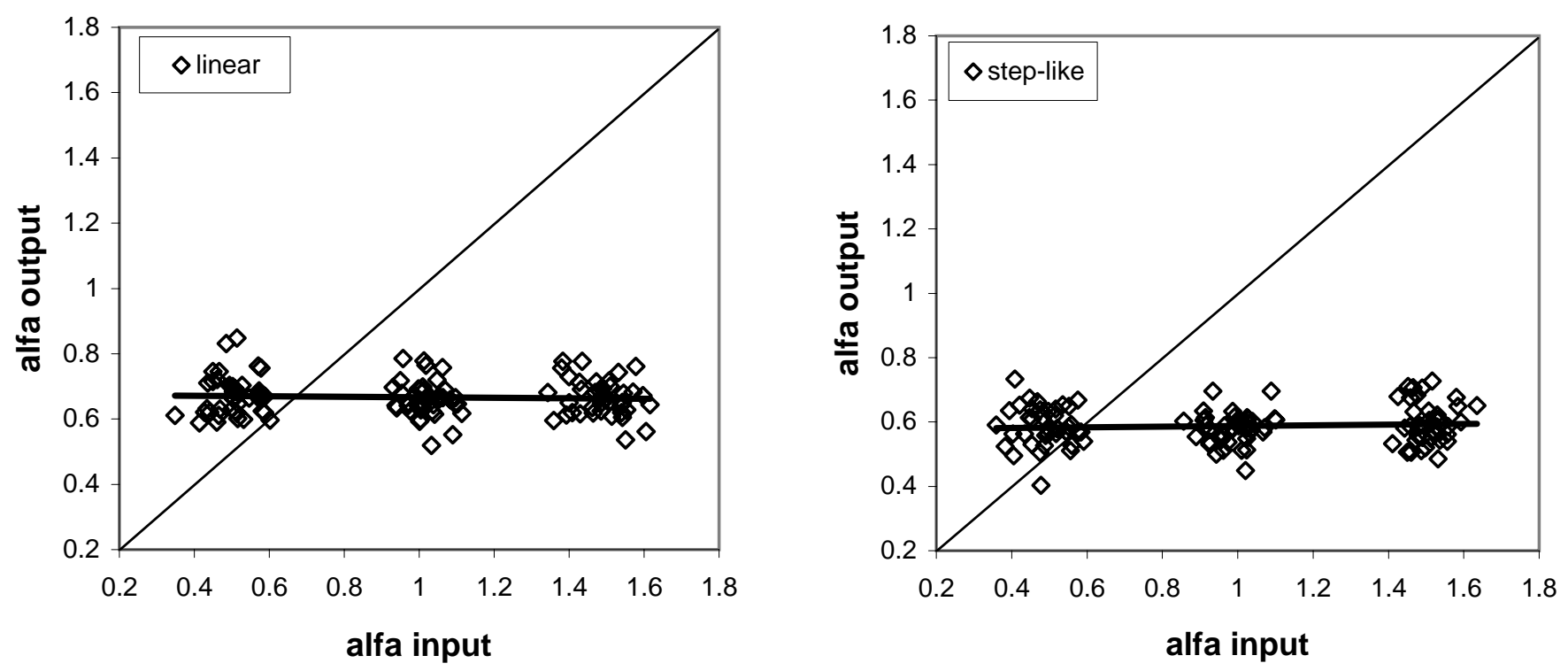

Fig. 7. DFA-calculated vs. expected $\alpha$ exponents for magnitude RSS. All the simulated series were interpolated, resampled by 0.07 day $^{-1}$ frequency and their magnitude series were computed. After, DFA was performed to assess the $\alpha$ values.

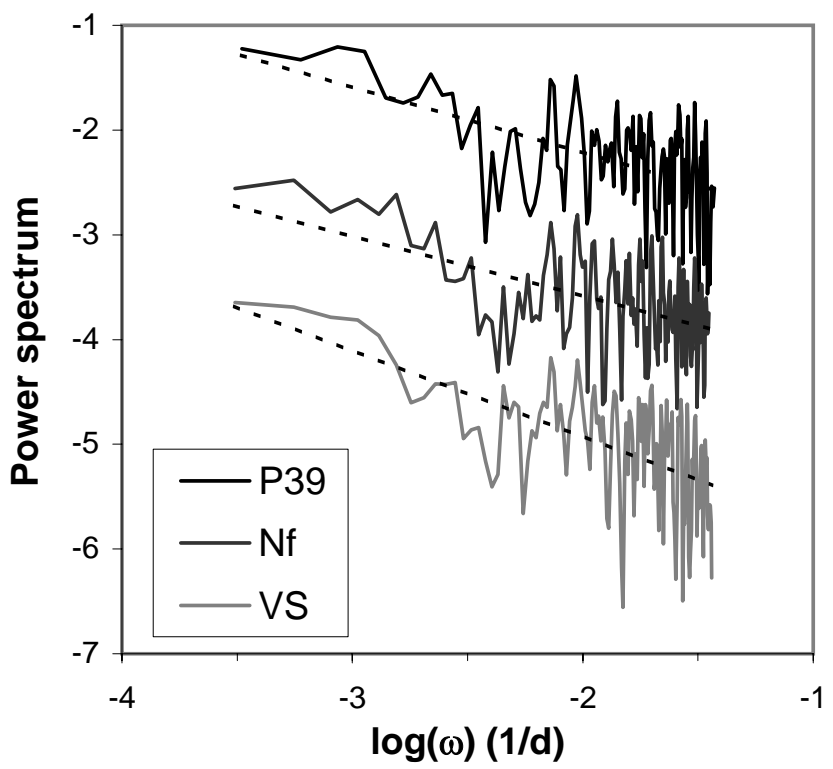

Fig. 8. Power spectra of unevenly sampled isotope series from sites P39, VS and Nf, computed by the Lomb periodogram method. The dotted lines are the best fit of a power law distribution of the spectral frequencies. Lomb periodograms were computed by the source routine available at http://www.physionet.org/physiotools/ wfdb/psd/lomb.c.

the case of nonlinearity estimations by TR test, we used the Constrained Randomization technique for unevenly sampled series (Sect. 3.3.2), which preserves both autocorrelation and PDF of the original signal. It means that the related null hypothesis is a linear stochastic series, regardless its PDF, and any non-Gaussianity of the original series cannot affect the result of the test. We also recall that the TR test does not systematically reject the linear null hypothesis in some kinds of very noisy signals (see Sect. 3.1 and Schreiber and Schmitz, 1997), nevertheless a positive result can be considered a sufficient condition to assess nonlinearity even in these difficult cases.

\section{Application to geochemical time series}

We applied the described statistical methods to: (i) the $\mathrm{He}$ isotope time series from all five monitored sites (see Sect. 2) and (ii) some significant time series of the $\mathrm{He}$ and $\mathrm{CO}_{2}$ concentrations from selected sites. Among the available He isotopic time series, the longest ones (sites VS, P39, and Nf) are discussed in Sect. 4.1, while the shortest (Fd and $\mathrm{St}$ ) are considered in Sect. 4.2. Finally, selected time series of the $\mathrm{He}$ and $\mathrm{CO}_{2}$ chemical concentrations are discussed in Sect. 4.3.

After computing the Lomb periodogram and TR test of the unevenly sampled GS, we interpolated the series and applied the DFA method to the RGS. In order to evaluate the achieved results, they were always compared to the statistics coming from the surrogate series analyses. First-order DFA (simply defined as DFA in this paper) was normally used, although we also performed calculations with second- and third-order DFA (DFA-2 and DFA-3, respectively). Finally, we computed the magnitude series of the RGS, and the related DFA and scaling exponent of magnitude RGS to asses the extent of nonlinearity. 

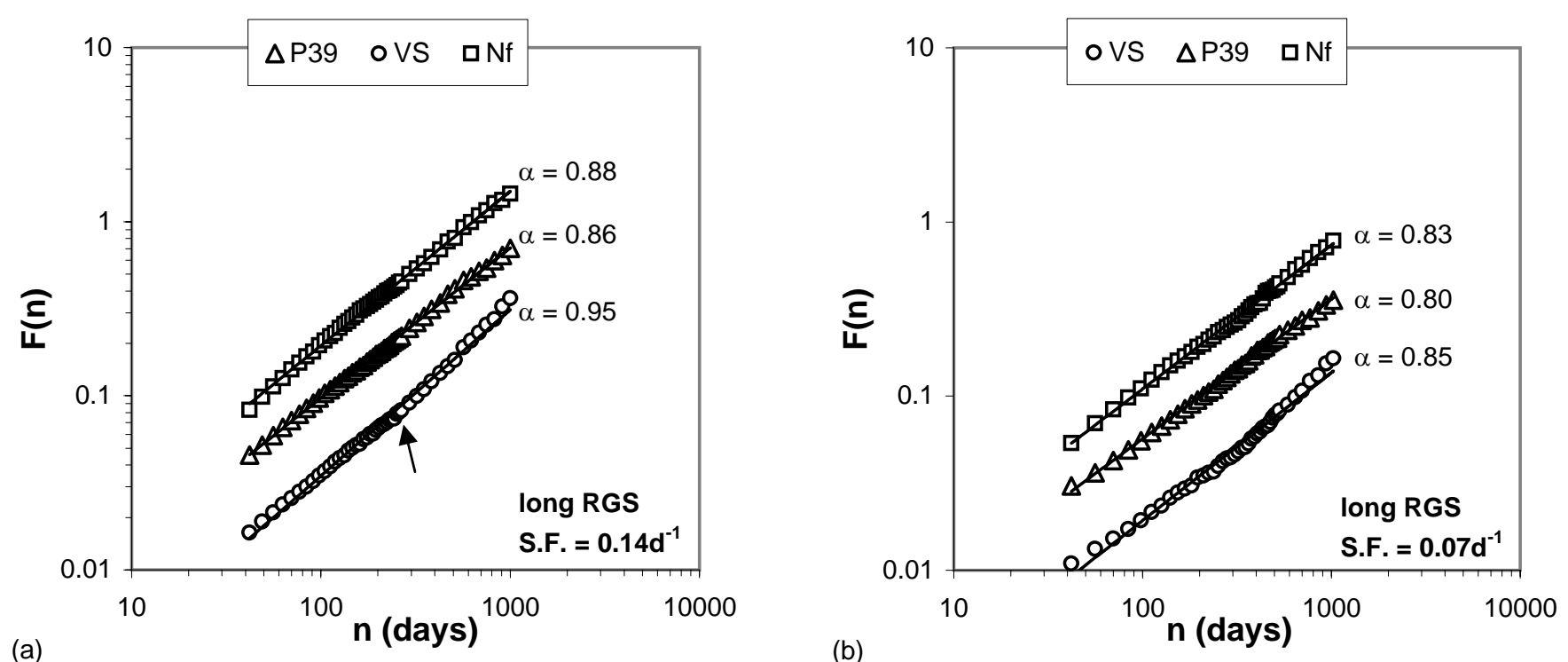

Fig. 9. Log-log plot of the fluctuation function $F(n)$ vs. time scale $n$, for the RGS from Nf, VS and P39 sites. The $F(n)$ functions of the sites have been shifted by a factor 2 for clarity, being partly overlapped. The series were step-like interpolated and resampled by frequency (S.F. in graphs) of: (a) $0.14 \mathrm{day}^{-1}$; (b) $0.07 \mathrm{day}^{-1}$. The arrow highlights the probable presence of a crossover.

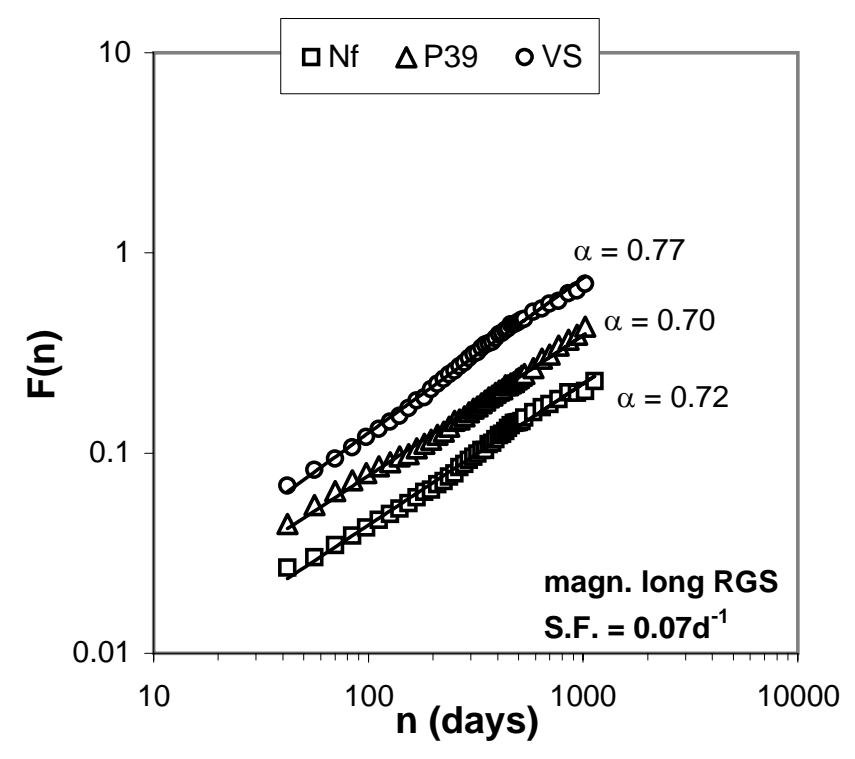

Fig. 10. Log-log plot of $F(n)$ vs. $n$, for the magnitude RGS from Nf, VS and P39 sites. The $F(n)$ functions of the sites have been shifted by a factor 2 for clarity. The series were step-like interpolated and resampled by 0.07 day $^{-1}$ frequency.

\subsection{Long-He-isotope time series}

In Fig. 8, we show the power spectrum of unevenly sampled isotope GS from sites P39, Nf, and VS, computed by the Lomb periodogram. All exhibit a somewhat power-law distribution of the spectral frequencies, suggesting the occurrence of long-range correlations. Table 1 gives the values of
Table 1. Scaling $(\alpha)$ and time reversibility $(\gamma)$ of isotopic GS.

\begin{tabular}{crrrrr}
\hline Site & $\alpha^{\mathrm{a}}$ & $\gamma^{\mathrm{b}}$ & Site & $\alpha^{\mathrm{a}}$ & $\gamma^{\mathrm{b}}$ \\
\hline VS & 0.89 & -0.97 & Fd & 0.99 & -1.8 \\
P39 & 0.81 & 0.46 & St & 1.05 & -2.2 \\
Nf & 0.78 & -0.51 & & & \\
\hline
\end{tabular}

a computed by fitting power spectrum by using Eqs. (1), (6), after calculating the spectrum by the Lomb periodogram method on unevenly sampled GS (see text).

b computed by means of Eq. (2) on unevenly sampled GS (see text). Nonlinearity is proved for $\gamma$ values higher or lower than $0.05<\gamma<0.05$ (see text).

the scaling exponents $\alpha$, as computed by Eq. (6). They fall between 0.78 and 0.89 , close to the signature of long-range correlated, flicker-noise signals. Site VS exhibits the highest scaling exponent, whereas the strength of the correlation seems to be slightly weaker at site Nf, the most distant from Mt. Etna (Fig. 1). In Table 1, we also provide the estimated values of $\gamma$, as achieved by performing the TR tests on the same GS (Eq. 2). When compared to the range defined by the surrogate series (Table 1), the values are sharply different. We thus reject the null hypothesis of linearity with a 95\% confidence, suggesting that all three series exhibit nonlinear properties. Again, site VS seems to display the largest asymmetry under time reversal.

As a following step, we attempt to quantify the recognized properties of scaling and nonlinearity by using DFA. Figure 9a displays the fluctuation function for the longestisotope RGS from sites P39, Nf, and VS, interpolated using the step-like method and resampled at a frequency of 0.14 day $^{-1}$. The portion of $[F(n)]$ for timescales shorter than 


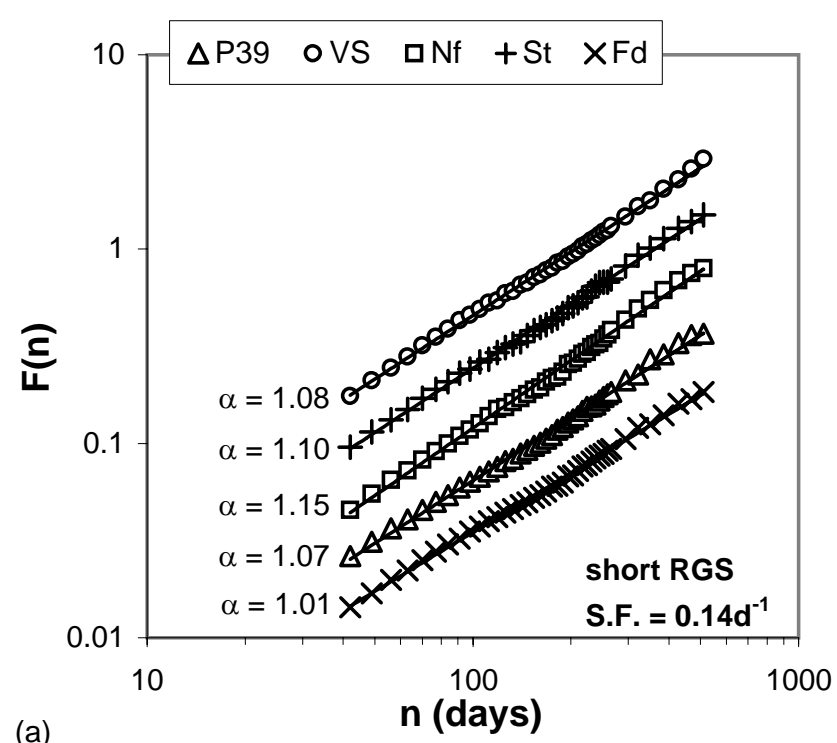

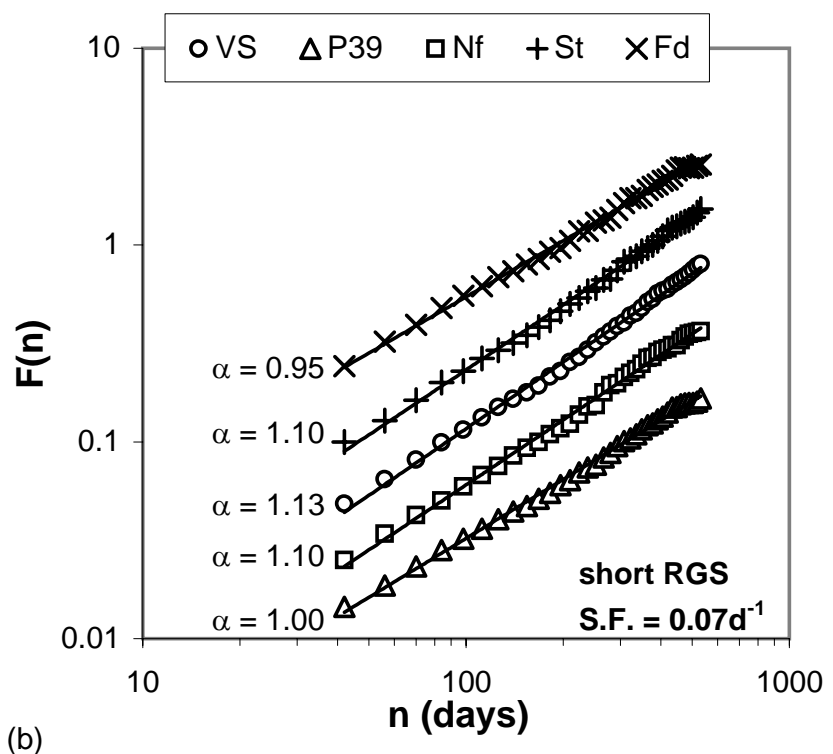

(b)

Fig. 11. Log-log plot of $F(n)$ vs. $n$, for the RGS from all five sites. Before DFA, the RGS from Nf, P39 and VS were shortened so as to cover the same time period of the series from Fd and St (see text). The $F(n)$ functions of the sites have been shifted by a factor 2 for clarity. The series were step-like interpolated and resampled by frequency of: (a) $0.14 \mathrm{day}^{-1}$; (b) $0.07 \mathrm{day}^{-1}$.

42 days is not shown due to the above-discussed deflection at short timescales. All time series show power-law distribution of the second-order fluctuation statistics. They closely resemble flicker-noise signals because displaying long-range correlations with scaling exponents that approach unity. Site VS exhibits the highest scaling exponent, whereas P39 and $\mathrm{Nf}$ give slightly lower exponents. It is noteworthy that the $\alpha$ values are in very good agreement with those coming from the Lomb periodogram. As assessed on the basis of the surrogate analysis (see Fig. 5), this result suggests that the adopted interpolation schemes do not significantly affect the statistics of our GS at the investigated time scales. The results did not change significantly when the RGS were resampled at 0.07 day $^{-1}$, although the differences among the sites become smaller (Fig. 9b). It is also noteworthy that a slight crossover appears to be present at a timescale of around 300 days in the VS function, which increases the correlation exponent by about 0.2 units (from 0.88 to 1.08 ) from the short to long timescales. This could hint any differences in the underlying dynamics of the system between short and long timescales. We recall that similar increases in correlation were revealed by DFA of magnetic data from the summit of Mt. Etna, although the timescales were very different $(\sim 12$ days; Currenti et al., 2005b).

Interesting results were also obtained when trying to quantify the extent of nonlinearity by using the magnitude series of the three sites. When using a sampling frequency 0.14 day $^{-1}$, applying DFA to the magnitude RGS yielded bilogarithm plots of $F(n)$ vs. $n$ of poor quality, as already confirmed for RSS. In contrast, reliable estimates of the exponents were obtained using $0.07 \mathrm{day}^{-1}$ sampling frequency and the step-like interpolation (Fig. 10). In these conditions, $\alpha$ values were in the range $0.7-0.8$, clearly above the values obtained for monofractal RSS managed under the same conditions $(\alpha \cong 0.58)$. In accordance with the results from the TR tests, the above $\alpha$ values confirm that all the RGS exhibit significant degrees of multifractality and nonlinearity (Table 1).

Finally, we also applied DFA-2 and DFA-3 to the longisotope RGS in an attempt to identify polynomial trends (Hu et al., 2001). The slopes of $\log [F(n)]$ functions of the highorder DFA (not shown) did not differ greatly from those of first-order DFA, apart from the above-mentioned underestimation at short timescales that greatly restricts the reliable portion of the fluctuation function. It can be concluded that significant polynomial trends are not present in the RGS.

\subsection{Short isotope series and temporal variations of scaling}

The fluctuation functions of the short isotopic signals from sites $\mathrm{St}$ and $\mathrm{Fd}$ also exhibit long-range correlations, with exponents close to typical flicker-noise signals (Fig. 11). However, the correlations appear to be slightly stronger than those assessed by the analysis of the VS, P39, and Nf series, showing scaling exponents of 1.0 to 1.1 . Recalling that the isotope GS from St and Fd cover the period since 2001, the differences in correlations between long and short series is an attractive result that can be attributed to two main mechanisms: (i) the effect of shorter signals in the estimation of the scaling exponent; and (ii) the different behavior of the volcanic system between the periods 1996-2001 and 2001-2006. The 


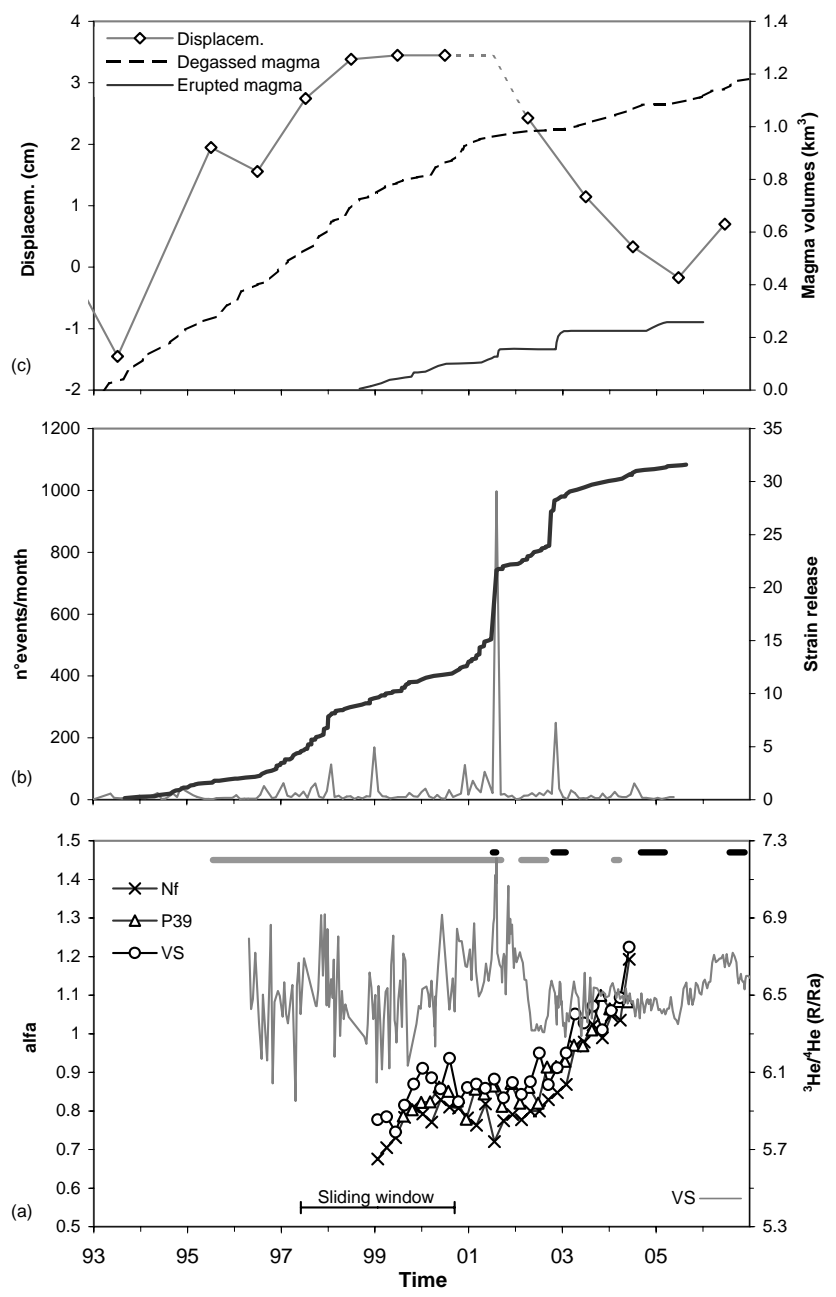

Fig. 12. Variations of some geochemical and geophysical parameters during the period 1993-2006. (a) unhandled He isotopic time series from VS site (grey curve), and local $\alpha$ exponents of the isotopic GS (symbols). The time length of the sliding window for calculating local $\alpha$ is displayed (see text). The horizontal lines on the top indicate the main eruptive phases: summit activities (thick grey segments) and flank eruptions of 2001, 2002-2003, 2004 and 2006 (black segments); (b) seismicity expressed as both monthly rate of events and strain release; (c) one-year mean vertical displacement of the volcanic cone, and cumulative erupted and degassed magma (see Allard et al., 2006; Neri et al., 2009, and their references for sources of geophysical data).

reliability tests of scaling exponent estimation on RSS having similar length revealed only a slight decrease in precision (standard errors of about $0.1 \alpha$ units), although their accuracies are similar. Despite the larger error bars due to the shortness of the series, their comparable accuracies exclude the former of the two above hypotheses. In order to check the second hypothesis, we studied the signals from VS, P39, and $\mathrm{Nf}$ after removing the portion from 1996 to 2000. This made the data directly comparable to the St and Fd series in terms of both statistical significance and covered time period. As evident in Fig. 11, the results for the shortened VS, P39, and $\mathrm{Nf}$ series are very similar to those for St and Fd, irrespective of the selected resampling frequency. In fact, the series from all five sites display long-range correlation, with scaling exponents significantly higher than those of the long series.

The differences between the long and shortened series emphasize a very probable change in the behavior of the natural system over time. In order to investigate this hypothesis, DFA was coupled to a sliding-window method (see Lapenna et al., 2004, for a case study applied to geochemical data). Basically, a window that was half the length of the long series was moved along the series whilst DFA was performed to measure the temporal changes in the scaling exponent. Applying this calculation to the three long series from VS, $\mathrm{P} 39$, and $\mathrm{Nf}$ revealed a progressive change in the correlation (Fig. 12a) that exceeds the estimated error bars. The $\alpha$ values start to increase in all the sites around 2002, when most of the sliding window includes the portion of the series after the 2001 and 2002-2003 eruptions. Due to the long time period covered by the sliding window, it is not possible to better localize the onset of the change. Although narrower sliding windows could be in theory used to increase the temporal resolution, the statistical reliability becomes too low. Anyway, the increase of scaling lasts throughout the entire second half of the series, with $\alpha$ exponents changing from $0.8-0.9$ up to 1.2. The change seems also to become sharper in the final piece of the series (Fig. 12a).

Finally, we studied the nonlinearity of the signals from St and Fd, by firstly performing the TR test. The $\gamma$ values allowed to reject the null hypothesis of linear stochastic processes, revealing fingerprint of nonlinearity conditions (Table 1). We analyzed the magnitude series related to the short signals from St and Fd, and to the shortened series from VS, NF, and P39. As stated in Sect. 3.3.1, we only used the 0.07day $^{-1}$ sampling frequency, but the quality of the computed power law functions was still not very high (Fig. 13). Although less confident, the obtained results have practically the same significance as those derived from longer signals. In fact, the scaling exponents of the sites range from 0.7 to 0.8 , except for the lower value of the $\mathrm{Nf}$ series. Comparing these values with 0.58 of monofractal RSS again reveals the appreciable extent of multifractality and nonlinearity of the GS, in accordance with the TR analyses.

\subsection{Time series of $\mathrm{He}$ and $\mathrm{CO}_{2}$ concentration}

The results presented above clearly indicate that the temporal variations in the He isotope composition exhibited both longrange correlation and nonlinearity. Such properties are somehow associated with the release of volatiles from magma, since isotope signals are strictly linked to the magma ascent and degassing (see Sect. 2). Likewise, temporal variations in noble gas and $\mathrm{CO}_{2}$ concentrations have been frequently linked to volatile degassing due to magma decompression (Nuccio and Paonita, 2001; Paonita, 2005), with a 


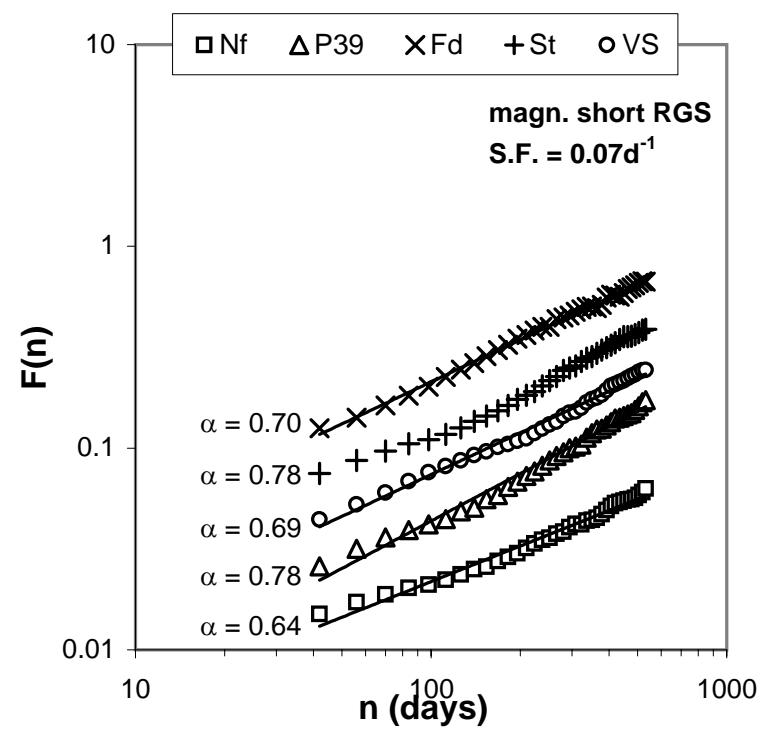

Fig. 13. Log-log plot of $F(n)$ vs. $n$, for the magnitude RGS from St, Fd and shortened VS, Nf and P39 signals. The $F(n)$ functions of the sites have been shifted for clarity.

recent focus on possible nonequilibrium effects (Paonita and Martelli, 2006). On this basis, we investigated the time series of chemical composition with two main aims: (i) to elucidate whether the concentration of volatiles shows temporal variations with flicker-noise dynamics (similar to the He isotope signals), and (ii) to understand how secondary nonmagmatic processes can affect the correlation and nonlinear statistics.

We selected two types of chemical series, one related to the magmatic system, and the other mainly affected by shallow secondary processes. We already stated that discharged gases from $\mathrm{Nf}$ are poorly affected by selective dissolution into aquifers, and thus the temporal variation of the He concentration is sufficiently representative of the true magmatic degassing signal. We then selected the He concentration time series of Nf for performing DFA. On the other hand, Caracausi et al. (2003b) inferred that temporal variations in the chemical composition of the gases from the VS vent showed the largest variations over time, due to the effects of selective dissolution in shallow aquifers (see Sect. 2). In fact, because the not-condensable fraction of magmatic gases is basically dominated by $\mathrm{CO}_{2}(>98 \mathrm{~mol} \%)$, the observed fluctuations in the $\mathrm{CO}_{2}$ concentration at VS (from 30 to $80 \mathrm{~mol} \%$ ) cannot be caused by magma degassing. Being coupled to opposing variations in the $\mathrm{He}, \mathrm{Ne}$, and $\mathrm{CH}_{4}$ concentrations, they fit a model of dissolution-driven fractionation in aquifers (Caracausi et al., 2003b). The same model can also be used to correct the He content with respect to the effects of dissolutiondriven fractionation, so as to restore the original magmatic signal. Therefore, we get (i) two time series carrying information about the magmatic system, namely the He concentration of $\mathrm{Nf}$ and the corrected He concentration of VS; and
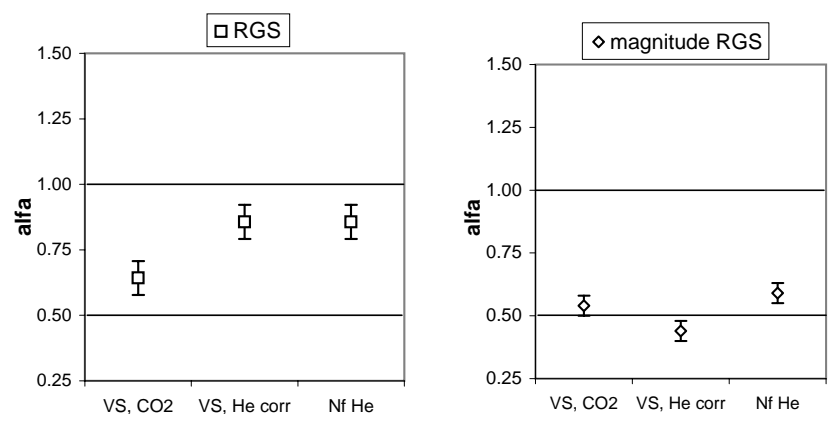

Fig. 14. Values of $\alpha$ exponent for the chemical RGS and related magnitude series. Labels in abscises are: "VS $\mathrm{CO}_{2}$ " the $\mathrm{CO}_{2}$ concentration series of VS site; "VS $\mathrm{He}$ corr." the He concentration series of VS site, once filtered out the dissolution effects; " $\mathrm{Nf}_{\mathrm{He}}$ " the $\mathrm{He}$ concentration series of site Nf. The calculated exponents have been corrected by the estimated accuracy errors (see Sect. 3.3) hence they can be directly compared to the theoretical values of flicker and white noise (solid horizontal lines).

(ii) one time series mainly affected by the shallow processes of dissolution-driven fractionation (the $\mathrm{CO}_{2}$-concentration time series of VS). We did not consider the uncorrected $\mathrm{He}$ concentration of VS due to the presence of coupling effects from both the magmatic and shallow systems.

Figure 14 displays the scaling exponent of the above three series as calculated for the isotopic GS. The He concentration RGS of both Nf and VS (the "magmatic" series) indubitably displayed long-range correlations, in accordance with the results from He isotope series. Indeed, it is understandable that the chemical and isotope series - both carrying direct information of the magmatic system - displayed similar correlations. The value of $\alpha$ was comparable to that for the longisotope series, and slightly lower than that for the shorter series. On the other hand, the TR test on the same two series does not highlight any significant time asymmetry and, accordingly, the calculated value of the exponent for the magnitude of these RGS essentially matched the range defined for white-noise RSS. These results indicate monofractality and linearity of these series, in contrast to those obtained for the magnitude isotope series.

DFA of the $\mathrm{CO}_{2}$ concentration RGS of site VS (the aquifer-controlled series) provided further information. The scaling exponent of this series was close to the values for white-noise RSS (Fig. 14), highlighting lack of long-range correlation and random temporal fluctuations. Calculation of the $\alpha$ value of the corresponding magnitude series (Fig. 14), again yielded white-noise-like values and absence of nonlinearity. Since the secondary process of dissolution essentially controls the series (see Sect. 2), we suggest that the shallow nonmagmatic dynamics produces neither long-range correlation nor nonlinearity. Therefore, the shallow system where magmatic gases selectively dissolve in groundwater seems to exhibit different dynamic features which do not involve feedback mechanisms. 


\section{Inferences on dynamics of magma degassing and relations with volcanic activity}

The results of DFA on isotopic GS indicate a clear powerlaw distribution of the second-order fluctuation function, that highlights the presence of long-range correlations. Coherently, the Lomb spectrum is power-law correlated too. When compared to signals with known correlation properties, the estimated values of the scaling exponent, close to unity, allow to recognize the isotopic GS as nearly flicker-noise or $1 / f$ signals. It means that the temporal fluctuations of the $\mathrm{He}$ isotope composition exhibit a fractal, power-law distribution, without any characteristic time scale (i.e., scale invariance). Finally, the significant asymmetry under time reversal, coupled to the long-range correlations of the related magnitude series, provide evidences of multifractality and nonlinearity in all the isotopic GS.

The efforts of recognizing the scale invariance and nonlinearity in the isotopic series arise from the fact that, as recalled in Sect. 1, signals showing such peculiar characteristics are generally produced by natural systems working in self-organized critical conditions (Sornette and Sornette, 1989; Turcotte, 1992). In addition to scale invariance and nonlinearity in time-dependent variables, such systems can be also featured by a power-law falloff in PDF of some observable. The isotopic GSs of this study exhibit the two former signatures of SOC (namely, the scaling exponents close to one in the second-order fluctuation statistics and the nonlinearity of the series), while they show near-Gaussian PDF (see Sect. 3.3). Nevertheless, pink-noise time series with Gaussian PDF have been already associated to SOC systems (Vattay and Harons, 1994), and likewise non-Gaussian distributions have not implied necessarily self-organized criticality (Krommes and Ottaviani, 1999). In addition, some models working under SOC have not produced pink-noise time series (Jaeger et al., 1989). Power-law PDF can also regard only the time fluctuations of a signal rather than the signal itself (e.g. some financial data, electrostatic plasma potentials, air humidity series). To sum up, there is not an universally accepted criterion to recognize a system as one working under SOC. In accordance with Hergarten (2002), we can reason about systems having different degrees of SOC, which move from weak criticality with one single condition, up to strong SOC where all the features are present.

It should be also noted that the physical explanation of flicker-noise signals, as well as their ubiquitous presence in nature, was in fact one of the main motivations for the initial proposal of the SOC concepts (Bak et al., 1987). In accordance with the SOC idea, systems where many interacting components are driven away from equilibrium by an external force, exhibit strongly nonlinear threshold behaviors and release their energy via events whose size are powerlaw distributed (i.e. they have no characteristic scale). Lacking a characteristic length will cause lacking a characteristic time, which will induce a power-law behavior into the frequency spectrum. Therefore, the temporal fingerprint of these systems will consists in long-range correlated and nonlinear $1 / f$-like signals. Since the seminal work of Bak et al. (1987), this type of signals have been considered as evidences of underlying SOC dynamics in thousands of articles on the most different topics. Accordingly, here we suggest that the system that generates our geochemical signals works under SOC conditions.

The importance of discovering this intriguing property arises when recalling certain features of the isotope signals. As stated in Sect. 2, the isotope temporal fluctuations have been related to magma ascent and degassing, by ruling out significant contributions of crustal and/or atmospheric sources. Therefore, the revealed long-range correlation and nonlinearity appear to be directly related to the process of volatile degassing from magma. Because systems in conditions of SOC involve the basic requirements mentioned in Sect. 1, we can use the SOC paradigm to constrain the dynamic conditions that characterize magma degassing and the physico-chemical aspects of such processes which can produce the SOC signatures. On this basis, we suggest that magma degassing has to be controlled by slowly driven, interaction-controlled and threshold conditions, and that nonequilibrium conditions must dominate throughout the process, or at least at the most important stages. $\mathrm{Nu}$ cleation and coalescence among bubbles are probably the best candidates in providing the above conditions, because they exhibit threshold and feedback behaviors, respectively (Gaonach'h et al., 1996; Blower et al., 2002), both of which are required to produce complex signatures. On this ground, the competition between slowly growing supersaturation (the driving force) and bubble nucleation, growth and coalescence (the threshold and nonlinear processes) in ascending magma could allow the system to evolve far from thermodynamic equilibrium, at least in the initial phase of degassing. Quantitative models will need to assess the actual effects of these processes on $\mathrm{He}$ isotope signals.

It is noteworthy that the isotope signal from the remote $\mathrm{Nf}$ site shows similar scaling and nonlinearity of the other emissions, hence the same processes must modulate this time series. Based on the established link between the SOC signature and degassing, our results suggest that volatile-rich magma bodies are present in this area, reinforcing the findings of Caracausi et al. (2003b). On the other hand, the nonlinearity that involves the He isotopic signature of degassing, seems to be absent in the chemical signature (see Sect. 4.3). Unless it has been lost in chemical time series as a result of the selective dissolution effects in aquifers (although the latter are small or largely filtered out), this condition would interestingly imply that the dynamics of isotopic and chemical degassing are different.

Our results have also revealed variations in the strength of the long-range correlations of the isotopic GS over time, as raised by the calculated changes of the scaling exponents (Sect. 4.2 and Fig. 12a). During the investigated period, 
Mt. Etna also showed several changes in volcanic activity, which can be convincingly related to the above changes of correlation. Since 1993, very large volumes of magma fed the degassing of Mt. Etna, as estimated on the basis of $\mathrm{SO}_{2}$ released by the volcanic plume (Allard et al., 2006). Such volumes largely exceed those actually erupted in the same period (Fig. 12c), suggesting a mechanism of storage of degassed melts in the Etnean system. It is in agreement with the results of deformation measurements, which put into evidence a general inflation trend of the volcano in the period 1993-1998, followed by a few years of minor deformation (Neri et al., 2009). The onset of the 2001 eruption marked a clear change in the rate of magma accumulation, suggesting an abrupt decrease of the deep magma supply to the feeding system (Fig. 12c). Accordingly, the eruption also marks the start of a deflationary trend (Fig. 12c). It can be reasonably thought that Mt. Etna progressively increased its eruptive potential until the 2001 eruption, whereas the subsequent energetic activities allowed the volcano to discharge its energy in excess. We recall that the 2001 and 2002-2003 paroxysms were extremely vigorous (Behncke and Neri, 2003; Andronico et al., 2005), with the latter culminating in exceptional explosive activity, whereas explosiveness and spattering were almost absent during the 2004 effusion (Burton et al., 2005; Corsaro and Miraglia, 2005) and weak during the 2006 eruption (Behncke et al., 2007). The growing levels of activity of Mt. Etna until 2002 were also highlighted by the rate of release of seismic energy from the system (Fig. 12b), while such rate showed a unambiguous decrease after the 2002 2003 eruptions.

From a geochemical point of view, the different behavior of the system before and after the 2001 and 2002-2003 events is already recognizable on the unhandled isotope GS (Fig. 12a): large and high-frequency fluctuations clearly occur until 2001, whereas the signal is much more smoothed during the following years. In terms of scaling, there is a progressive variation from a persistent, flicker-like signal up to 2002, towards Brownian, Gaussian-integrated series later (Fig. 12a). It suggests that temporal changes in the dynamic behavior of the system occurred, although they are not easy to explain from a deterministic point of view. Once more, we can use concepts from the SOC paradigm to carry out some considerations. If high levels of activity imply severe nonequilibrium conditions, the system should exhibit typical self-organized critical dynamics and nonlinearity during these periods. Thus, the increase in the exponent after the 2002 could sign a change from a complex behavior toward Brownian dynamics, typical of less-energetic periods. Although it is not the goal of this paper to model the magmatic process capable to produce isotope signals having the required time-dependent scaling features, we recall that several approaches are emerging to describe time-dependent nonlinear dynamics in magma chambers (see Sparks, 2003, for a review). In addition to a number of physical variables (e.g. heat loss and crystallization, frequency and volume of eruptions), such models put into evidence the effects of the varying extent of internal overpressure in destabilizing magma chambers, which critically depends on the rate of magma refilling. On this ground, we remind that the 2001 and 2002-2003 eruptions displayed the involvement of a different magma with respect to the typical one feeding Etnean activity, being richer in volatiles and more explosive, and among the most primitive erupted in the past centuries (Corsaro et al., 2007). The massive input of this new magma in the Etnean feeding system would have caused the overpressure responsible for the geochemical and geophysical anomalies prior to the 2001 event, as well as the changes of scaling properties. It is matter of discussion if the scaling and nonlinearity of our signals is a consequence of the atypical features of such magma, particularly linked to its high content of volatiles, or alternatively if any massive magmatic refilling would produce similar conditions.

\section{Summary and conclusion}

Several years of geochemical monitoring of the gas emissions from sites around Mt. Etna have made available long time series of data, which have allowed us to apply statistical tools to detect the scaling and nonlinear properties of these signals. We have used power spectrum, Lomb periodogram, time reversibility and detrended fluctuation analysis to investigate how time-series data of the isotopic and chemical composition of $\mathrm{He}$ and $\mathrm{CO}_{2}$ are linked to the magmatic activity of Mt. Etna. The results clearly indicate that the temporal fluctuations of the He isotope composition exhibit no characteristic scales (i.e., scale invariance) and long-range correlations with a fractal power-law distribution. Irrespective of their location in the volcano edifice, all the sites provide isotope signals marked by power-law exponents close to 1 , which are typical of dynamic systems in a self-organized critical state. Moreover, both TR test and DFA of the magnitude series, derived from the isotope signals, reveal remarkable degrees of nonlinearity, which are normally found in systems exhibiting complex behavior and feedback mechanisms among interacting processes. Such properties put constraints on the intrinsic and hidden dynamics of the system, suggesting that it works in conditions of self-organized criticality. Evidence of longrange correlation also emerges by DFA of He concentration time series, once the effects of chemical fractionation due to selective dissolution in aquifers have been basically filtered out. In this case, the nonlinearity appears however to be lost.

Based on geochemical evidences, previous works have strictly linked the fluctuations of the investigated $\mathrm{He}$ isotopic signals to the pre-eruptive degassing of volatiles from ascending and decompressing magma. Thus, our previous result that the system generating the signals works under criticality conditions allows us to conclude that the process of magma degassing is able to yield the typical signature of self-organized complex dynamics. By using the conceptual 
framework of SOC, degassing has hence to be controlled by slowly driven, interaction-dominated and threshold conditions. Degassing in effect involves: (i) bubble nucleation and/or detachment, which display evident threshold behaviors, (ii) bubble coalescence, that is able to exhibit nonlinear cascading regimes of bubble growth, and (iii) recharging, decompression and growing supersaturation which act as driving forces. As a result, it would not be surprising if any degassing indicator displays the SOC signature.

SOC in magma dynamics has been revealed by statistical analyses of several types of observables, ranging from the occurrence of eruptive events to acoustic emissions, ground deformations, geomagnetic anomalies, volcanic tremors, and seismicity (Grasso and Bachèlery, 1995; Vinciguerra and Barbano, 2000; Diodati et al., 2000, 2004; Kostantinou and Schlindwein, 2002; Telesca et al., 2002; Vinciguerra, 2002; Currenti et al., 2005a, b; Walter et al., 2005). It is noteworthy that in most of these cases, the complex behavior can also be attributed to magma interaction with the stress and strain fields of the volcanic structure through which the magma migrates (see Grasso and Bachèlery, 1995). Hence, such interaction can also affect magma degassing by cause-and-effect mechanisms, and it could transfer the SOC signature to the degassing indicators. Future research should be aimed to assess whether the pre-eruptive degassing process is really able to exhibit its own complexity or whether is simply a consequence of the mechanisms of magma migration.

On the other hand, the analysis of $\mathrm{CO}_{2}$ concentration time series - which are directly affected by the shallow processes of chemical equilibrium between gas and groundwater - reveals the absence of both long-range correlation and nonlinearity. The temporal fluctuations of the signals are essentially random, so that the shallow system where the volcanic gases selectively dissolve into groundwaters does not appear to be governed by complexity conditions. Although the network of rock fractures influences the fluid flow and the extent of gaswater chemical interactions, and complex behaviors could be accordingly expected, our results appear to contradict this guess. This should be kept in mind when linking the complexity of fluid-flow-induced observables with the fractality of geological structures.

Finally, our results suggest temporal changes in the correlation strength of the He isotope signals. In particular, we found that the scaling exponent progressively increased since 2002, meaning that the strength of the correlation increased from a flicker-noise type toward higher values. Despite the low time resolution of our analysis, it unambiguously displays that the 2001 and 2002-2003 eruptions signed a dramatic change in the dynamic behavior of the Etnean system. This outcome agrees with the hints coming from observing several geophysical signals, which recognize two periods marked by sharply different behavior of the volcanic system: (i) prior to the 2001 eruption, showing growing levels of activity and energy; (ii) subsequent to the 2002-2003 eruptions, marked by a decrease of activity. During the former period, the feeding system of Mt. Etna was massively refilled by a primitive and volatile-rich magma, never observed in the volcano. Accordingly, Mt. Etna volcanism between 20012003 was among the most energetic in recent times, whereas subsequent eruptions were much less vigorous. On this basis, the observed transition of Mt. Etna system from typical SOC toward Brownian-like dynamics seems to be strictly linked to the state of activity of the volcano. In detail, the typical behavior of system at a critical state would become manifest during periods of strong unrest of the volcano due to massive input of volatile-rich magmas in the plumbing system. Longer monitoring will be required to prove this attractive hypothesis.

Acknowledgements. I thank my colleagues of the Etna Group at INGV-Pa (A. Caracausi, M. Martelli, P. M. Nuccio, and A. Rizzo), whose efforts produced the large data set needed for the statistical analyses. The use of English was checked by English Science Editing. Comments of D. Schertzer and two anonymous reviewers really improved the manuscript. This article is dedicated to my wife Simona.

Edited by: D. Schertzer

Reviewed by: two anonymous referees

\section{References}

Allard, P., Behncke, B., D'Amico, S., Neri, M., and Gambino, S.: Mount Etna 1993- 2005: Anatomy of an evolving eruptive cycle, Earth Sci. Rev., 78, 85-114, doi:10.1016/j.earscirev.2006.04.002, 2006.

Andronico, D., Branca, S., Calvari, S., Burton, M., Caltabiano, T., Corsaro, R. A., Del Carlo, P., Garfì, G., Lodato, L., Miraglia, L., Murè, F., Neri, M., Pecora, E., Pompilio, M., Salerno, G., and Spampinato, L.: A multi-disciplinary study of the 2002-03 Etna eruption: Insight into a complex plumbing system, Bull. Volcanol., 67, 314-330, 2005.

Ashkenazy, Y., Ivanov, C. I., Havlin, S., Peng, C.-K., Goldberger, A. L., and Stanley, H. E.: Magnitude and sign correlations in heartbeat fluctuations, Phys. Rev. Lett., 86, 1900-1903, 2001.

Ashkenazy, Y., Havlin, S., Ivanov, C. I., Peng, C.-K., SchulteFrohlinde, V., and Stanley, H. E.: Magnitude and sign scaling in power-law correlated time series, Physica A, 323, 19-41, 2003.

Bak, P., Tang, C., and Wiesenfeld, K.: Self-organized criticality: An explanation of the 1/f noise, Phys. Rev. Lett., 59, 381-384, 1987.

Barunik, J. and Kristoufek, L.: On Hurst exponent estimation under heavy-tailed distributions, Physica A, 389-18, 3844-3855, 2010.

Behncke, B. and Neri, M.: The July-August 2001 eruption of Mt. Etna (Sicily), Bull. Volcanol., 65, 461-476, 2003.

Behncke, B., Calvari, S., Neri, M., and Giammanco, S.: 2006 summit eruptions of Mount Etna (Italy): rock and lava avalanches resulting from interaction of basaltic magma with external water, Geophys. Res. Abstr., 9, EGU 02524, 2007.

Blower, J. D., Keating, J. P., Mader, H. M., and Phillips, J. C.: The evolution of bubble size distributions in volcanic eruptions, J. Volcanol. Geotherm. Res., 120, 1-23, 2002. 
Burton, M. R., Neri, M., Andronico, D., Branca, S., Caltabiano, T., Calvari, S., Corsaro, R. A., Del Carlo, P., Lanzafame, P., Lodato, L., Miraglia, L., Salerno, G., and Spampinato, L.: Etna 2004-2005: An archetype for geodynamicallycontrolled effusive eruptions, Geophys. Res. Lett., 32, L09303, doi:10.1029/2005GL022527, 2005.

Caracausi, A., Favara, R., Giammanco, S., Italiano, F., Paonita, A., Pecoraino, G., Rizzo, A., and Nuccio, P. M.: Mount Etna: Geochemical signals of magma ascent and unusually extensive plumbing system, Geophys. Res. Lett., 30(2), 1057, doi:10.1029/2002GL015463, 2003a.

Caracausi, A., Italiano, F., Paonita, A., Rizzo, A., and Nuccio, P. M.: Evidence of deep magma degassing and ascent by geochemistry of peripheral gas emissions at Mount Etna (Italy): Assessment of the magmatic reservoir pressure, J. Geophys. Res., 108(B10), 2463, doi:10.1029/2002JB002095, 2003b.

Centamore, C., Montalto, A., and Patanè, G.: Self-similarity and scaling relations for microearthquakes at Mt. Etna volcano (Italy), Phys. Earth Planet. Inter., 103, 165-177, 1997.

Cernelc, M., Suki, B., Reinmann, B., Hall, G. L., and Frey, U.: Correlation properties of tidal volume and end-tidal $\mathrm{O}_{2}$ and $\mathrm{CO}_{2}$ concentrations in healthy infants, J. Appl. Physiol., 92, 18171827,2002

Chen, Z., Ivanov, P. C., Hu, K., and Stanley, H. E.: Effect of nonstationarities on detrended fluctuation analysis, Phys. Rev. E, 65, 041107, doi:10.1103/PhysRevE.65.041107, 2002.

Coronado, A. V. and Carpena, P.: Size Effects on Correlation Measures, J. Biolog. Phys., 31, 121-133, 2005.

Corsaro, R. A. and Miraglia, L.: Dynamics of 2004-2005 Mt. Etna effusive eruption as inferred from petrologic monitoring, Geophys. Res. Lett., 32, L13302, doi:10.1029/2005GL022347, 2005.

Corsaro, R. A., Miraglia, L., and Pompilio, M.: Petrologic evidence of a complex plumbing system feeding the July-August 2001 eruption of Mt. Etna, Sicily, Italy, Bull. Volcanol., 69, 401-421, 2007.

Currenti, G., Del Negro, C., Lapenna, V., and Telesca, L.: Fluctuation analysis of the hourly time variabilità of volcanic-magnetic signals recorded at Mt. Etna volcano, Sicily (Italy), Chaos Solitons Fractals, 23, 1921-1929, 2005a.

Currenti, G., Del Negro, C., Lapenna, V., and Telesca, L.: Scaling characteristics of local geomagnetic field and seismicity at Etna volcano and their dynamics in relation to the eruptive activity, Earth Planet. Sci. Lett., 235, 96-106, 2005 b.

Currenti, G., Del Negro, C., Johnston, M., and Sasai, Y.: Close temporal correspondence between geomagnetic anomalies and earthquakes during the 2002-2003 eruption of Etna volcano, J. Geophys. Res., 112, B09103, doi:10.1029/2007JB005029, 2007.

Diks, C., van Houwelingen, J. C., Takens, F., and DeGoede, J.: Reversibility as a criterion for discriminating time series, Phys. Lett. A, 201, 221-235, 1995.

Diodati, P., Bak, P., and Marchesini, F.: Acustic emissions at the Stromboli volcano: scaling laws and seismic activity, Earth Planet. Sci. Lett., 182, 235-258, 2000.

Diodati, P., Piazza, S., and Marchesini, F.: Acoustic emission from periodically perturbed systems: SOC and predictability, Physica A, 340, 598-606, 2004.

Gaonac'h, H., Lovejoy, S., Stix, J., and Scherzter, D.: A scaling growth model for bubbles in basaltic lava flows, Earth Planet. Sci. Lett., 139, 395-409, 1996.
Gliozzi, M., Brinkmann, W., Rath, C., Papadakis, I. E., Negoro, H., and Scheingraber, H.: On the nature of X-ray variability in Ark 564, Astron. Astrophys., 391, 875-886, 2002.

Goldberger, A. L., Amaral, L. A. N., Glass, L., Hausdorff, J. M., Ivanov, P. Ch., Mark, R. G., Mietus, J. E., Moody, G. B., Peng, C. K., and Stanley, H. E.: PhysioBank, PhysioToolkit, and PhysioNet: Components of a New Research Resource for Complex Physiologic Signals, Circulation, 101(23), e215-e220, 2000.

Govindan, R. B., Wilson, J. D., Preißl, H., Eswaran, H., Campbell, J. Q., and Lowery, C. L.: Detrended fluctuation analysis of short datasets: an application to fetal cardiac data, Physica D, 226, 23-31, 2007.

Grasso, J. R. and Bachèlery, P.: Hierarchical organization as a diagnostic approach to volcano mechanics: Validation on Pito de la Fournaise, Geophys. Res. Lett., 22, 2897-2900, 1995.

Hegger, R., Kantz, H., and Schreiber, T.: Practical implementation of nonlinear time series methods: The TISEAN package, Chaos, 9, 413-440, 1999.

Hergarten, S.: Self-organized Criticality in Earth Systems, Springer-Verlag Berlin Heidelberg New York, 274 pp., 2002.

Hu, K., Ivanov, P. I., Chen, Z., Carpenna, P., and Stanley, H. E.: Effect of trends on detrended fluctuation analysis, Phys. Rev. E, 64, 011114, doi:10.1103/PhysRevE.64.011114, 2001.

Jaeger, H. M., Liu, C.-H., and Nagel, S. R.: Relaxation at the Angle of Repose, Phys. Rev. Lett., 62, 40-43, 1989.

Kantelhardt, J. W., Konscielny-Bunde, E., Rego, H. H. A., Havlin, S., and Bunde, A.: Detecting long-range correlations with detrended fluctuation analysis, Physica A, 295, 441-454, 2001.

Kantelhardt, J. W., Zschiegner, S. A., Kosciliny-Bunde, E., Bunde, A., Pavlin, S., and Stanley, H. E.: Multifractal detrended fluctuation analysis of nonstationary time series, Physica A, 316, 78114, 2002.

Kantz, H. and Schreiber, T.: Nonlinear time series analysis, 2nd ed., 369 pp., Cambridge University Press, Cambridge, 2004.

Kostantinou, K. I. and Schlindwein, V.: Nature, wavefield properties and source mechanism of volcanic tremor: a review, J. Volcanol. Geotherm. Res., 119, 161-187, 2002.

Krommes, J. A. and Ottaviani, M.: Long-time tails do not necessarily imply self-organized criticality or the breakdown of the standard transport paradigm, Phys. Plasmas, 6, 3731-3735, 1999.

Lapenna, V., Martinelli, G., and Tedesca, L.: Long-range correlation analysis of earthquake-related geochemical variations recoreded in Central Italy, Chaos Solitons Fractals, 21, 491-500, 2004.

Lomb, N. R.: Least-squares frequency analysis of unequally spaced data, Astrophys. Space Sci., 39, 447-462, 1976.

Lovejoy, S. and Schertzer, D.: Scaling and multifractal fields in the solid earth and topography, Nonlin. Processes Geophys., 14, 465-502, doi:10.5194/npg-14-465-2007, 2007a.

Lovejoy, S. and Schertzer, D.: Scale, scaling and multifractals in geophysics: twenty years on, in: Nonlinear dynamics in Geosciences, edited by: Tsonis, A. A. and Elsner, J., Springer, 311337, $2007 b$.

Marty, B., Trull, T., Lussiez, P., Basile, I., and Tanguy, J. C.: He, $\mathrm{Ar}, \mathrm{O}, \mathrm{Sr}$ and $\mathrm{Nd}$ isotope constraints on the origin and evolution of Mount Etna magmatism, Earth Planet. Sci. Lett., 126, 23-39, 1994.

Nagarajan, R.: Surrogate analysis of volatility series from longrange correlated noise, Physica A, 374, 281-288, 2007. 
Newman, M. E. J. and Eble, G. J.: Power spectra of extinction in the fossil record, Proc. Biol. Sci., 266(1425), 1267, doi:10.1098/rspb.1999.0773, 1999.

Nuccio, P. M. and Paonita, A.: Magmatic degassing of multicomponent vapors and assessment of magma depth: Application to Vulcano Island (Italy), Earth Planet. Sci. Lett., 193, 467-481, 2001.

Paonita, A.: Noble gas solubility in silicate melts: a review of experimentation and theory, and implications regarding magma degassing processes, A. Geophys., 48, 647-669, 2005.

Paonita, A. and Martelli, M.: Magma dynamics at mid-ocean ridges by noble gas kinetic fractionation: assessment of magmatic ascent rates, Earth Planet. Sci. Lett., 241, 138-158, 2006.

Patanè, G., La Delfa, S., and Tanguy, J.-C.: Volcanism and mantlecrust evolution: the Etna case, Earth Planet. Sci. Lett., 241, 831843, 2006.

Peng, C.-K., Havlin, S., Stanley, H. E., and Goldberger, A. L.: Quantification of scaling exponents and crossover phenomena in nonstationary heartbeat time series, Chaos, 5, 82-87, 1995.

Porta, A., Guzzetti, S., Montano, N., Gnecchi-Ruscone, T., Furlan, R., and Malliani, A.: Time Reversibility in Short-Term Heart Period Variability, Comput. Cardiol., 33, 77-80, 2006.

Rizzo, A., Caracausi, A., Favara, R., Martelli, M., Paonita, A., Paternoster, M., Nuccio, P. M., and Rosciglione, A.: New insights into magma dynamics during last two eruptions of Mount Etna as inferred by geochemical monitoring from 2002 to 2005, Geochem. Geophys. Geosyst., 7, Q06008, doi:10.1029/2005GC001175, 2006.

Schmitz, A. and Schreiber, T.: Testing for nonlinearity in unevenly sampled time series, Phys. Rev. E, 59, 4044-4047, 1999.

Schreiber, T. and Schmitz, A.: On the discrimination power of measures for nonlinearity in a time series, Phys. Rev. E, 55, 54435449, 1997.
Sornette, A. and Sornette, D.: Self-organized criticality and earthquakes, Europhys. Lett., 9, 197-201, 1989.

Sparks, R. S. J.: Forecasting volcanic eruptions, Earth Planet. Sci. Lett., 210, 1-15, 2003.

Subba Rao, T. and Gabr, M. M.: An introduction to bispectral analysis and bilinear time series models, in: Lecture notes in statistics, 24, Springer-Verlag, Berlin, Heidelberg, New York, 1984.

Taqqu, M. S., Teverovsky, V., and Willinger, W.: Estimators for long-range dependence: an empirical study, Fractals, 3(4), 785788, 1995.

Telesca, L., Cuomo, V., Lapenna, V., and Macchiato, M.: Timeclustering analysis of volcanic occurrence sequences, Phys. Earth Planet. Inter., 131, 47-62, 2002.

Theiler, J., Eubank, S., Longtin, A., Galdrikian, B., and Farmer, J. D.: Testing for nonlinearity in time series: The method of surrogate data, Physica D, 58, 77-94, 1992.

Turcotte, D. L.: Fractal and chaos in geology and geophysics, Cambridge University Press, New York, 1992.

Vattay, G. and Harons, A.: Scaling behavior in daily air humidity fluctuations, Physic. Rev. Lett., 73(5), 768-771, 1994.

Vinciguerra, S.: Damage mechanics preceding the SeptemberOctober 1989 flank eruption at Mt. Etna volcano inferred by seismic scaling exponents, J. Volcanol. Geotherm. Res., 113, 391397, 2002.

Vinciguerra, S. and Barbano, S.: Clustering of seismicity at Mount Etna, Phys. Chem. Earth (A), 25, 699-703, 2000.

Walter, T. R., Acocella, V., Neri, M., and Amelung, F.: Feedback processes between magmatic events and flank movement at Mount Etna (Italy) during the 2002-2003 eruption, J. Geophys. Res., 110, B10205, doi:10.1029/2005JB003688, 2005. 